\title{
Biomimetic sponges improve muscle structure and function following volumetric muscle loss
}

Gabriel Haas ${ }^{\dagger}$, Andrew Dunn', Josh Madsen, Peter Genovese, Andrew Lin, Hannah Chauvin, Jeffrey Au, Allison Paoli, and Koyal Garg

${ }^{+}$Authors contributed equally

Department of Biomedical Engineering, Parks College of Engineering, Aviation, And Technology, Saint Louis University

Address correspondence to

Koyal Garg, PhD

Assistant Professor

Department of Biomedical Engineering

Parks College of Engineering, Aviation, and Technology

3507 Lindell Blvd

St. Louis, MO 63103

Phone: 314.977.1434

Email: koyal.garg@slu.edu 


\begin{abstract}
Skeletal muscle is inept in regenerating after traumatic injuries such as volumetric muscle loss (VML) due to significant loss of basal lamina and the resident satellite cells. Currently, there are no approved therapies for the treatment of muscle tissue following trauma. In this study, biomimetic sponges composed of gelatin, collagen, laminin-111, and FK-506 were used for the treatment of VML in a rodent model. We observed that biomimetic sponge treatment improved muscle structure and function while modulating inflammation and limiting the extent of fibrotic tissue deposition. Specifically, sponge treatment increased the total number of myofibers, type 2B fiber cross-sectional area, myosin: collagen ratio, myofibers with central nuclei, and peak isometric torque compared to untreated VML injured muscles. As an acellular scaffold, biomimetic sponges provide a promising “off-the-shelf” clinical therapy for VML.
\end{abstract}




\section{Introduction}

Skeletal muscle is endowed with a remarkable capacity for regeneration, but "volumetric muscle loss" (VML) presents a unique challenge due to the unrecoverable loss of basal lamina and resident stem cell population that results in chronic functional impairment and disability [14]. Autologous tissue grafts from an uninjured site are currently used to treat VML injuries in the clinic [5]. However, this complicated surgical approach can cause donor site morbidity, infection, and has been reported to result in graft failure in $4-7 \%$ of cases [6, 7]. Physical therapy is typically recommended to VML patients to strengthen the remaining muscle mass, but it is unable to facilitate appreciable muscle regeneration within the site of injury [8]. Therefore, there is a clinical need to develop bioengineered therapies for muscle regeneration and reconstruction following VML.

The extracellular matrix (ECM) forms an ideal microenvironment for cell survival and activity. The ECM not only provides a framework for structural and mechanical support but also sequesters cytokines and growth factors to orchestrate cell migration, proliferation, and differentiation $[9,10]$. As a result, acellular ECM scaffolds have been extensively used for the treatment of skeletal muscle injuries. However, there is mounting evidence to suggest that decellularized ECM scaffolds do not support muscle regeneration and remodel into a dense collagenous scar [11-14]. This inability to promote muscle regeneration is primarily attributed to insufficient satellite cell proliferation and activity in the defect region $[1,15,16]$. Furthermore, these scaffolds are unable to modulate the overwhelming immune response that dysregulates the muscle regeneration process [14]. We have previously demonstrated that heightened and prolonged immune response to musculoskeletal trauma impairs regeneration [17]. As early as three days post-VML, both innate and adaptive immune cells can be observed in the VML defect, and their persistent presence impairs muscle regeneration by extending the inflammatory phase and delaying the repair and remodeling phase.

The fungal macrolide FK-506 (Tacrolimus) is an FDA approved immunosuppressant that disrupts signaling events mediated by calcineurin in T lymphocytes [18]. By inhibiting calcineurin, FK-506 prevents dephosphorylation of nuclear factor of activated T cells (NFAT), which reduces the production of IL-2, a cytokine that promotes autocrine T-cell development and proliferation. Besides T-cells, FK-506 can also modulate the pro-inflammatory cytokine 
production by dendritic cells and macrophages [19, 20]. Since FK-506 selectively reduces proinflammatory cytokine production, it can potentially promote $\mathrm{T}$-helper $2\left(\mathrm{~T}_{\mathrm{H}} 2\right)$ versus $\mathrm{T}$ helper 1 ( $\left.\mathrm{T}_{\mathrm{H}} 1\right)$ responses $[21,22]$. In a recent study, intraperitoneal (i.p.) injection of FK-506 (1 mg/kg) at the time of injury in rats with composite muscle-bone trauma attenuated the immune response in the VML injured skeletal muscle. At day three post-injury and FK-506 injection, total T-cell numbers in VML injured muscle reduced by $67 \%$, helper T-cells specifically by $51 \%$, and macrophages by $22 \%$ [23]. However, the administration of FK-506 alone did not result in functional muscle regeneration. In a subsequent study, when FK-506 was injected i.p. and VML defect was treated with autologous minced muscle grafts in a porcine model, muscle regeneration and function was enhanced [24]. Taken together, these studies suggest that a combination of regenerative and immunomodulatory therapy is essential for functional muscle regeneration.

In a previous study, we demonstrated that a unique blend of gelatin, collagen, and laminin-111 in a porous sponge-like scaffold supports the infiltration of satellite cells, promotes myogenic protein expression, and myofiber regeneration in a murine model of VML [25]. In this study, we incorporated FK-506 in the biomimetic sponges to enhance their immunomodulatory properties. We hypothesize that biomimetic sponges will support functional recovery in a rat model of VML by stimulating regeneration and limiting the extent of inflammation and fibrosis.

\section{Materials and Methods}

\section{Preparation of collagen/gelatin/laminin/FK506 sponge}

A 3 wt \% porcine skin gelatin (Sigma-Aldrich) solution was prepared in DI water and heated to $60^{\circ} \mathrm{C}$. After the gelatin had completely dissolved, the solution was allowed to cool to $50^{\circ} \mathrm{C}$. EDC $(20 \mathrm{mM})$ was added to the solution which was combined with rat tail collagen I (Gibco, $3 \mathrm{mg} / \mathrm{mL}$ ) at a 70:30 gelatin : collagen ratio in a tube. Laminin (LM)-111 (Trevigen) and FK506 (Abcam) were added to the solution at final concentrations of $50 \mu \mathrm{g} / \mathrm{mL}$ and $25 \mu \mathrm{M}$, respectively. The final solution was then aliquoted into a 48 -well plate at $700 \mu \mathrm{L} /$ well. The well plate was placed in a $100 \%$ methanol bath, allowing the sponges to gel at $4^{\circ} \mathrm{C}$ for $30 \mathrm{~min}$, followed by overnight freezing at $-8^{\circ} \mathrm{C}$. The well plate was then removed from the bath and moved to $\mathrm{a}-80^{\circ} \mathrm{C}$ freezer for 48 hours and subsequently lyophilized for at least 12 hours. The day before surgery, the sponges were disinfected via a 5-minute incubation in 200-proof pure 
ethanol, which was followed by two 5-minute rinses, and an overnight rinse in sterile $1 \mathrm{x}$ phosphate-buffered saline (Gibco).

\section{Implantation of sponges into a VML model}

This work was conducted in compliance with the Animal Welfare Act, the implementing Animal Welfare Regulations, and in accordance with the principles of the Guide for the Care and Use of Laboratory Animals. All animal procedures were approved by the Saint Louis University's Institutional Animal Care and Use Committee.

Male Lewis rats (10-12 weeks old) were purchased from Charles Laboratory and housed in a vivarium accredited by the Association for Assessment and Accreditation of Laboratory Animal Care International and provided with food and water ad libitum. The animals were weighed prior to surgery and anesthetized using $2.5 \%$ isoflurane. The surgical site was aseptically prepared, and sustained release buprenorphine $(1 \mathrm{mg} / \mathrm{kg})$ was injected in the nape of the neck prior to the procedure. A lateral incision was made through the skin to reveal the tibialis anterior (TA), and the skin was separated from the musculature by blunt dissection. A metal plate was inserted underneath the TA muscle, and a 6-mm punch biopsy was performed to remove approximately $\sim 20 \%$ of the muscle mass. The biopsy was removed and weighed for consistency. A subset of injured animals received biomimetic sponges while the other subset was left untreated. A 6-mm sponge disk was used to treat the TA. Bleeding was controlled with light pressure, and the skin incision was closed with simple interrupted skin staples. The animals were

allowed to recover for 7,14 , or 28 days and euthanized via exsanguination followed by cervical dislocation. TA muscles were weighed upon collection $(n=9-17$ muscles, and $5-9$ animals per group) and processed for histological and biomolecular analyses. The surgery was performed bilaterally, keeping the treatment subsets consistent between both legs.

\section{$\underline{\text { Histology }}$}

TA muscles were cut in half at the defect site, and the upper portion was frozen in 2methylbutane (Fisher Scientific) super-cooled in liquid nitrogen for 10 seconds. The muscles were mounted on stubs using OCT, and transverse cross-sections $(15 \mu \mathrm{m})$ were cryosectioned from the area where the original surgical defect was made. Cross-sections were stained with hematoxylin and eosin (H\&E), collagen 1 (1:100; ab34710; Abcam, Cambridge MA), sarcomeric 
myosin (1:50; MF20; Developmental Studies Hybridoma Bank), laminin (1:100; ab11575; Abcam; Cambridge MA), CD68 (1:50; MCA341R; AbD Serotec, Raleigh, NC), nuclei (DAPI; 1:100; Invitrogen), CD3 (1:100; ab5690; Abcam, Cambridge MA), CD31 (1:100; R\&D systems), $\alpha$-bungarotoxin (1:100; Invitrogen). Appropriate fluorochrome-conjugated secondary antibodies (1:100; Invitrogen) were used as described previously [1, 3, 5]. Images were captured at $10 \times$ and $20 \times$ magnification using a Zeiss Axiocam microscope. Slides were scanned to obtain composite images of the entire muscle section using Olympus BX614S (Saint Louis University) and NanoZoomer 2.0 HT (Washington University in Saint Louis).

Full-size muscle sections stained with H\&E were used to quantify the number of myofibers with centrally located nuclei at day 28 post-injury ( $\mathrm{n}=4$ muscles, $2-3$ animals). Fullsize muscle sections stained with myosin heavy chain (MHC) and collagen (COL) were used to quantify the MHC:COL ratio by $\%$ area ( $n=4-5$ muscles, $2-4$ animals). Images were opened in ImageJ (or Fiji) and the remaining defect area and remaining muscle tissue were divided into two separate images of the same size. The two images (i.e., one containing the defect area and the other containing the remaining healthy tissue), were separately analyzed by splitting the color channels, thresholding the MHC and COL to most accurately represent the stained area, and measuring the percentage of area positively stained by MHC and collagen. The \% area of MHC and collagen from the two images were added up, and the MHC: COL ratio was determined. The muscle sections were split into remaining muscle tissue and defect area in order to measure the collagen deposition accurately. The collagen tissue deposited in the defect showed different sensitivity to thresholding than the collagen in the remaining healthy tissue. When thresholding without splitting, low thresholding results in the defect area not being picked up, whereas high thresholding results in collagen filling the muscle fibers. Thresholding the defect region and the remaining healthy tissue separately allowed for accurate detection and measurement of collagen (Supplemental Figure 1).

Muscle cross-sections were stained using antibodies from Developmental Studies Hybridoma Bank (Iowa City, IA, USA) for fiber types 1 (1:20; BA.D5), 2A (1:50; SC.71), and 2B (1:20; BF.F3), as described previously [26]. A laminin counterstain (1:100; ab11575) served as the fiber outline. Unstained fibers were identified as type $2 \mathrm{X}$. A custom-designed image analysis MATLAB program was used for the quantification of myofiber cross-sectional area $(\mathrm{CSA} ; \mathrm{n}=4$ 
- 5 muscles, $3-4$ animals) and fiber type distribution ( $n=3-5$ muscles, $3-4$ animals). Briefly, the algorithm first thresholds the laminin channel, followed by nonlinear morphological transformations to delineate fiber boundaries and reduce noise. Area, circularity, and concavity filters are applied to identify myofibers. To avoid spatial variances in brightness, a color histogram is computed for each fiber, and areas corresponding to only a single channel are then compared to determine the primary fiber-type. If no color is dominant, the fiber is marked as $2 \mathrm{X}$.

\section{Flow Cytometry}

Cells were isolated from the entire TA muscle with the muscle defect ( $n=4-6$ muscles, $2-4$ animals) by enzymatic digestion as previously described $[17,23]$. Briefly, the TA was surgically isolated, and the mass was determined. Tissue was incubated with collagenase type II and dispase for $90 \mathrm{~min}$ at $37^{\circ} \mathrm{C}$. Cells were further released by gentle mechanical disruption and filtered through a $70 \mu \mathrm{m}$ cell strainer and subsequently through a $40 \mu \mathrm{m}$ cell strainer. Erythrocytes were lysed with ammonium-chloride-potassium lysing buffer, and cells were washed and resuspended in Roswell Park Memorial Institute (RPMI) medium. Viable cells were quantified using trypan blue exclusion and a hemocytometer. After quantification, cells were resuspended at $10^{6}$ cells $/ \mathrm{mL}$ in PBS containing $0.5 \% \mathrm{FBS}$ and $0.1 \%$ sodium azide. From the resuspension, the cells were incubated with anti-CD32 antibody (BD Biosciences 550271) to block Fc receptors and labeled with either cocktail monoclonal antibodies to identify macrophages or T lymphocytes. The macrophage cell cocktail included anti-CD11b (BD Biosciences 562102, clone WT.5), anti-CD86 (BD Biosciences 743211, clone 24F), and antiCD163 (Bio-Rad MCA342F, clone ED2). The T lymphocyte cell cocktail (BD Biosciences 558493 ) consisted of anti-CD3 (IF4), anti-CD4 (OX-35), and anti-CD8 $\alpha$ (OX-8). Samples were run on BD biosciences LSR II flow cytometer at the flow cytometry research core facility at Saint Louis University.

\section{$\underline{\text { Gene Expression }}$}

As described previously [5], RNA was isolated from snap-frozen cross-sections of TA muscle ( $\mathrm{n}=4-5$ muscles, $2-4$ animals) that was comprised of both the defect area and the remaining muscle mass (50 mg). RNA was extracted using Trizol LS reagent (Invitrogen) and purified using RNeasy mini kit (Qiagen). The yield of RNA was quantified using a NanoDrop 
spectrometer (NanoDrop Technologies) and optical density (OD) 260/280 ratios were determined. RNA (500 ng) was reverse transcribed into cDNA using the Super-Script III firststrand synthesis kit (Invitrogen). Custom designed primers (Sigma-Aldrich) with the sequences presented in Table 1 were used as myogenic and immunogenic markers. All primer sets have been synthesized by Sigma-Aldrich DNA oligos design tool. Aliquots $(2 \mu \mathrm{l})$ of cDNA were amplified with $200 \mathrm{nM}$ forward/reverse primers, SYBR GreenER (Invitrogen) in triplicate using a Bio-Rad CFX96 thermal cycler system (Bio-Rad). Nontemplate control and no reverse transcriptase controls were run for each reaction. Gene expression was normalized to $18 \mathrm{~S}$ (housekeeping gene) to determine the $\Delta \mathrm{CT}$ value. Expression levels for each mRNA transcript were determined by the $2^{-\Delta \Delta \mathrm{CT}}$ method by normalizing each group to uninjured cage control muscles.

Additionally, gene expression of immunogenic markers was measured using a PCR immunoarray, as described previously [17]. RNA isolated as described above and was converted into complementary DNA (cDNA) using $\mathrm{RT}^{2}$ First Strand Kit (Qiagen). An RT $\mathrm{R}^{2}$ Profiler PCR array (PARN052Z, SABiosciences) was employed to examine rat innate and adaptive immune responses. A CFX96 Real-Time System (Bio Rad) was used to determine gene expression. Data analysis was carried out either with the online $\mathrm{RT}^{2}$ Profiler PCR Array Data Analysis version 3.5 or expression levels for each mRNA transcript were determined by the $2^{-\Delta \mathrm{CT}}$ method. Gene expression in VML-injured muscle tissue was relative to expression in uninjured cage control muscle tissue and normalized to reference gene Rplp1 ( $n=4$ per group).

\section{Muscle Function Assessment}

In vivo functional testing of the anterior crural muscles $(n=7-10$ muscles, $4-6$ animals) was performed at 28 days post-injury using the methodology previously described [23]. Briefly, in vivo physiological properties were measured in anesthetized rats (isoflurane 1.5 2.0\%) using a dual-mode muscle lever system (Aurora Scientific, Inc., Mod. 305b). The skin was shaved, and an incision was made at the postero-lateral aspect of the ankle. The distal tendon of the gastrocnemius-soleus complex muscles was isolated and severed to prevent plantarflexion. Subcutaneous needle electrodes were inserted in the posterior compartment of the lower limb on each side of the common peroneal nerve. Optimal current $(30-40 \mathrm{~mA})$ was set with a series of 
twitches. Isometric tetanic contractions were elicited at $150 \mathrm{~Hz}(0.1 \mathrm{~ms}$ pulse width, $400 \mathrm{~ms}$ train) with the ankle at a right angle.

\section{$\underline{\text { Statistical analysis }}$}

Data are presented as a mean \pm standard error of the mean. Analysis and graphing of data were performed using GraphPad Prism 8 for Windows. A one-way or two-way analysis of variance was used when appropriate to determine if there was a significant interaction or main effect between variables. The Fisher's least significant difference post-hoc comparison was performed to identify significance with $\mathrm{p} \leq 0.05$. The PCR immunoarray data was analyzed using the non-parametric Kruskal-Wallis test followed by Dunn's post-hoc test to identify significance with $\mathrm{p} \leq 0.05$.

\section{Results}

\section{FK506 Release in vitro}

The amount of FK506 released from the sponges over 7 days is shown in Figure 1. The majority of the FK-506 loaded on to the sponges was released within the first 3 days, suggesting a burst release. At days 1 and 2, sponges loaded with $25 \mu \mathrm{M}$ of FK-506 released a significantly higher percentage of the drug compared to sponges loaded with 50 and $100 \mu \mathrm{M}$ of FK-506. On day 4, almost all of the FK-506 in the sponges had diffused out in vitro.

\section{Myogenic marker expression}

Gene expression of myogenic markers is shown in Figure 2. Pax7 and MyoD are markers associated with satellite cell activation and proliferation. Irrespective of treatment, Pax7 shows a transient decrease on day 14 followed by an increase on day 28. MyoD expression shows a linear increase between days 7 and 28 in both treated and untreated samples. Although not significant, myogenin expression is higher with sponge treatment on day 7. Similar to Pax7, it also shows a transient drop at day 14 followed by an increase at the day 28 time-point, but only in the sponge treated group. In the untreated group, myogenin expression remains relatively constant over 28 days. Embryonic myosin heavy chain (eMHC) maintains its expression over 14 days in the sponge treated group. However, it shows a sharp decline in the untreated groups. eMHC 
expression was higher in the sponge treated group compared to the untreated group at day 14 , but statistical significance was not reached.

A trend towards increased TGF- $\beta 1$ expression is observed with sponge treatment. Over time, both treated and untreated muscles show a sharp decline in TGF- $\beta 1$ expression. Collagen 1 gene expression is significantly reduced over time in the untreated muscles, but the expression is maintained in the sponge treated muscles.

\section{Flow cytometry}

Quantitative analysis of mononuclear cell infiltration was performed using flow cytometry at days 7, 14, and 28 post-injury. Cells isolated from the TA muscles were gated for T-lymphocytes $\left(\mathrm{CD}^{+}\right)$, followed by helper T-lymphocytes $\left(\mathrm{CD}^{+}\right)$and cytotoxic T-lymphocytes $\left(\mathrm{CD}^{+}\right)$. T-lymphocytes could be detected in the VML injured muscles as early as 7 days postinjury (Figure 3). At day 7, no differences in T-lymphocyte quantity were observed between untreated and treated VML injured muscles.

On day 14, the untreated muscles showed a significant decrease in both $\mathrm{CD}^{+}$and $\mathrm{CD}^{+}{ }^{+} \mathrm{CD} 4^{+} \mathrm{T}$-cells quantity but not $\mathrm{CD}^{+} \mathrm{CD}^{+} \mathrm{T}$-cells. In contrast, the quantity of both $\mathrm{CD} 3^{+}$ and $\mathrm{CD}^{+} \mathrm{CD}^{+} \mathrm{T}$-cells was maintained in the sponge treated muscles over 14 days. The sponge treated muscles showed a significant increase in $\mathrm{CD} 3^{+} \mathrm{CD} 8^{+} \mathrm{T}$-cell quantity at day 14 compared to day 7. A significantly higher quantity of $\mathrm{CD}^{+}, \mathrm{CD}^{+} \mathrm{CD}^{+}$, and $\mathrm{CD}^{+} \mathrm{CD}^{+} \mathrm{T}$-cells were observed in the sponge treated muscles compared to untreated muscles at day 14 . On day 28 , no significant differences were observed between treated and untreated muscles. Sponge treated muscles showed a significant decrease in $\mathrm{CD}^{+}, \mathrm{CD}^{+} \mathrm{CD}^{+}$, and $\mathrm{CD} 3^{+} \mathrm{CD} 8^{+} \mathrm{T}$-cell quantity compared to untreated muscles. Altogether, these data indicate that while in the untreated muscles the adaptive immune response subsides by day 14, an elevated and persistent T-cell response is observed in sponge treated muscles.

Mononuclear cells were also gated for $\mathrm{CD}_{1} 1 \mathrm{~b}^{+}$myeloid cells, followed by $\mathrm{CD} 86^{+}$proinflammatory M1-like cells and CD163+ anti-inflammatory M2-like cells (Figure 4). The

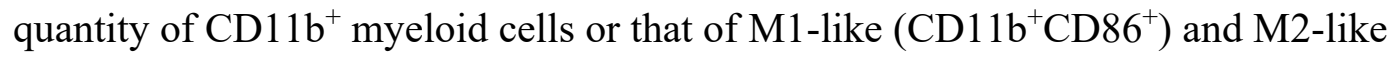
$\left(\mathrm{CD} 11 \mathrm{~b}^{+} \mathrm{CD} 163^{+}\right)$cells was not significantly different between treated and untreated groups on days 7,14 , and 28. A linear decrease in cell quantity was observed in both treated and untreated 
samples over 28 days. Overall, these data show mixed recruitment of pro- and anti-inflammatory macrophages in response to VML injury and sponge treatment.

\section{Modulation of the immune response post-VML}

A PCR array was used to quantify gene expression of markers associated with innate and adaptive immune responses (Figure 5). VML injury resulted in significant upregulation of tolllike receptors (TLR) -1, 2, 4, and 7 compared to cage controls. Treatment of VML with sponge matrix resulted in significant upregulation of TLR -5, 6, 9, in addition to TLRs-1, 2, 4, and 7 compared to cage controls. In untreated muscles, a trend towards increased gene expression of TLR-6 ( $p=0.0776)$ was observed. These results suggest that various TLRs are involved in the innate immune system's recognition and interaction with the implanted biomaterial [27, 28$]$. NOD-like receptors (NLR) are a class of pattern recognition receptors that are found in the cytosol. The gene expression of NLRP3 was significantly increased in the sponge treated muscles. The gene expression of NOD2 was trended towards an increase in sponge treated muscles ( $\mathrm{p}=0.0955)$ but was significantly higher in untreated muscles, compared to cage controls.

Myeloid differentiation factor 88 (MyD88) is an essential adaptor molecule for most TLRs, and it mediates the activation of nuclear factor $\kappa \mathrm{B}(\mathrm{NF}-\kappa \mathrm{B})$ signaling pathway. Irrespective of treatment, both VML injured muscles showed significantly higher expression of MyD88. Once activated via the NFKB signaling pathway, the innate immune system initiates the inflammatory response secreting chemokines and cytokines. The expression of interferon response factor (IRF)-3 and IRF7 was significantly higher in untreated VML injured muscles compared to cage controls. IRF3 gene expression trended higher in the treated muscles $(p=0.0624)$. As expected, VML injury caused increased gene expression of pro-inflammatory cytokines such as interleukin (IL)-1 $\beta$, IL-18, and IL-6, irrespective of treatment. Sponge treatment significantly increased the expression of interferon (IFN)- $\gamma$. The untreated VML injured muscles showed a significant increase in the expression of cytokine receptor IFN- $\gamma \mathrm{R} 1$. While the expression of IL-1R1 was significantly increased in both untreated and treated muscles, its expression was downregulated -2.13 fold in the sponge treated muscles. Sponge treatment resulted in a significantly higher expression of complement component 3 (C3) compared to cage controls. 
Several chemokines and their receptors were also significantly increased in response to VML injury such as CCL12, CCL5, and CCR5. Gene expression of both CXCL10 and CXCR3 that are involved in peripheral homing of T-cells was downregulated -2.01-fold with sponge treatment. The chemokine (C-X-C motif) ligand 10 (CXCL10) trended lower in sponge treated muscles (ANOVA p =0.0575). Cell adhesion proteins such as ITGAM and ICAM1 were increased in both treated and untreated muscles. The gene expression of ITGAM was significantly higher in untreated muscles and trended higher in treated muscles $(p=0.0624)$ compared to cage controls.

The JAK-STAT pathway is involved in the secretion of cytokines. The expression of STAT1 and STAT4 was significantly increased only in untreated muscles but trended higher in the treated muscles $(\mathrm{p}=0.0955)$. STAT3 gene expression was significantly increased in both untreated and treated muscles. In response to VML, a significant increase in Jak 2 was observed, irrespective of treatment. Although not statistically different, the gene expression of STAT6 and IL-13 was downregulated -23.04-fold and -2.25-fold, respectively.

Inflammatory factors released by innate immune cells can also recruit T-cells to the site of injury. VML injury resulted in significantly higher gene expression of CD8a, CD40, and CD86 compared to cage controls. Sponge treatment resulted in significantly lower CD8a compared to untreated muscles. Apoptosis associated genes such as Fas ligand (FasL) and caspase 1 were downregulated in sponge treated muscles -2.14 and -8.42-fold, respectively. FasL was significantly increased in untreated muscles compared to cage controls. Both untreated and treated VML injured muscles showed significantly higher expression of CD40 ligand (CD40LG) and CD80. The expression of MX2 was significantly higher in the untreated muscles only. MX2 was downregulated -11.52 fold in the sponge treated muscles compared to untreated muscles.

\section{Muscle Mass and Function}

TA muscle mass was significantly lower in the VML injured muscles irrespective of treatment on days 7, 14, and 28 post-injury compared to the uninjured controls (Figure 6A). A $>35 \%$ deficit in muscle mass was observed between uninjured controls and VML injured muscles over 28 days. No significant differences were observed between untreated and treated muscles at any time-points. 
The isometric strength of the anterior crural muscles was measured in vivo at 28 days post-injury. VML injury resulted in a $\sim 56-59 \%$ deficit in peak isometric torque production when analyzing raw force data and that normalized to body mass (Table 2). Sponge treatment significantly improved the torque production in VML injured muscles (Figure 6B-D). Compared to untreated muscles, biomimetic sponges improved muscle strength by $\sim 31-37 \%$ when analyzing raw force data and that normalized to body mass or TA muscle mass. Histological analysis of acetylcholine receptor clustering revealed no differences in muscle innervation between untreated and treated muscles (Figure 6E).

\section{Cellular infiltration, myofiber regeneration, and fibrosis}

Transverse cross-sections of TA muscles were stained with H\&E and are shown in Figure 7. At all three time-points, increased cellular infiltration $\left(\mathrm{DAPI}^{+}\right)$can be observed in the defect site. At Day 7, the implanted sponges can be identified in the VML defect. The sponges appear to support cellular infiltration and do not cause fibrotic capsule formation. By day 14, the sponges are no longer visible in the defect. On day 28, quantitative analysis revealed that there are significantly more myofibers with centrally located nuclei in treated muscles versus untreated muscles (Figure 7).

Muscle cross-sections were also stained with myosin heavy chain (MHC) and collagen (COL), as shown in Figure 8. On day 7, both untreated and treated muscles showed a large defect region filled with collagenous fibrotic tissue. The remodeled sponge could be identified in the defect. On day 14, the untreated muscles continued to show collagenous fibrotic tissue deposition. However, in the sponge treated muscles, the defect region appeared smaller with less fibrotic tissue. Several small diameter $\mathrm{MHC}^{+}$myofibers could also be identified in the defect region of the sponge treated muscles (Supplemental Figure 2). By day 28, the sponge treated muscles continued to show a smaller fibrotic region as well as increased presence of $\mathrm{MHC}^{+}$ myofibers in and around the defect region. Muscle regeneration and fibrosis was assessed by analyzing the ratio of myosin heavy chain to collagen (MHC: COL). The MHC: COL ratio was statistically similar between the untreated and sponge treated muscles on day 7. On day 14, sponge treated muscles trended towards an increased MHC: COL ratio $(\mathrm{p}=0.055)$, and on day 28, the sponge treated muscles showed statistically higher MHC: COL ratio compared to the untreated muscles. 
Qualitative analysis of the cellular infiltration showed that while $\mathrm{CD}^{+}$positive cells remained around the periphery, the $\mathrm{CD} 68^{+}$macrophages infiltrated the three-dimensional structure of the sponges (Figure 9). Both untreated and treated muscles supported CD31 $1^{+}$ endothelial cells and $\mathrm{SCA}^{+}$stem cell activity in the VML defect region.

\section{Cross-sectional area measurement}

The total number of myofibers in the cage control TA muscle cross-sections were determined to be $\sim 9865$. VML injury reduced the total number of myofibers in the TA muscle by $76.5 \%$ on day 14 . On both days 14 and 28 , sponge treatment significantly increased the total number of myofibers (treatment effect, $\mathrm{p}=0.0205$ ). The percentage improvement in the number of myofibers with sponge treatment was $77 \%$ and $82 \%$, on days 14 and 28 , respectively. The mean and median CSA was statistically similar between the untreated and treated muscles. The distribution of fiber CSA is shown in Figure 10. Sponge treatment significantly increased the number of small diameter myofibers $\left(<500 \mu \mathrm{m}^{2}\right)$ on both days 14 and 28 post-VML injury. The number of fibers in the CSA range of 500-999 $\mu \mathrm{m}^{2}$ and 1000-1499 $\mu^{2}$ were also significantly higher with sponge treatment on day 14 post-VML. The number of small diameter fibers $(<500$ $\mu \mathrm{m}^{2}$ ) showed a significant increase from day 14 to day 28 in the sponge treated groups. A simultaneous decline in the number of bigger fibers $\left(500-1500 \mu \mathrm{m}^{2}\right)$ was observed with sponge treatment on day 28 compared to day 14 .

Fiber type specific CSA analysis revealed no change in the percentage of either Type 1, 2A, 2B or $2 \mathrm{X}$ myofibers with VML injury (Figure 11). However, the mean CSA of fast-twitch glycolytic fibers (i.e., type $2 \mathrm{~B}$ and $2 \mathrm{X}$ ) was significantly lower with VML injury. Sponge treated muscles showed significantly larger CSA of type 2B myofibers compared to untreated muscles. Fiber type distribution analysis showed that sponge treatment significantly increased the number of small diameter fibers $\left(<500 \mu \mathrm{m}^{2}\right)$ in the slow oxidative fibers (i.e., type 1 and $2 \mathrm{~A}$ ), and largediameter myofibers $\left(>4000 \mu \mathrm{m}^{2}\right)$ in the fast-twitch type $2 \mathrm{~B}$ fibers.

\section{Discussion}

The most important finding of this study is that FK-506 loaded biomimetic sponges improve muscle structure and function in a rodent model of VML. Sponge treatment increased 
the total number of myofibers, type 2B fiber CSA, MHC: collagen ratio, and muscle function compared to untreated VML injured muscles. These results are encouraging, and we believe that biomimetic sponges may provide a promising "off-the-shelf" approach for VML that is worthy of clinical investigation.

An increase in the number of myofibers and type 2B fiber CSA may underlie the basis for improved muscle function with sponge treatment. To the best of our knowledge, a significant increase in myofiber number and muscle function has never been reported with the implantation of an acellular ECM scaffold in a full-thickness VML model. The use of decellularized scaffolds in VML injuries have shown conflicting results with studies reporting either no improvement $[15,29,30]$, exacerbated force deficits [31], or modest improvements in force production [32]. While scaffolds that contain myogenic stem cells have shown improvement in muscle regeneration and force production [5,33-35], their clinical translation is likely to be hindered by limited availability of autologous donor tissue and potential donor-site morbidity.

In this study, the biomimetic sponges were expected to provide both immunomodulatory as well as regenerative effects. The sponges contain an optimized ratio of ECM proteins such as gelatin, collagen, and laminin-111, and have supported myogenic activity in vitro and in vivo in a previous study [25]. Besides FK-506, immunomodulatory and anti-inflammatory effects can also be ascribed to ECM scaffolds [36]. While it is difficult to attribute specific immunomodulatory or regenerative effects to the different constituents of biomimetic sponges, our results suggest that their implantation modulated the VML microenvironment in a way that improved muscle structure and function. In support of this contention, the flow cytometry data showed that the implantation of sponges changes the temporal dynamics of the immune response in VML injured muscles. In contrast to untreated muscles where the presence of T-cells $\left(\mathrm{CD}^{+}, \mathrm{CD}^{+}\right)$ significantly reduces between days 7-14, the sponge treated muscles show maintained T-cell presence over 14 days post-injury. Interestingly, the percentage of $\mathrm{CD} 8^{+} \mathrm{T}$-cells peaks at day 14 in sponge treated muscles while it remains unchanged between days 7-28 in untreated muscles. At day 7 post-injury, while the percentage of infiltrating $\mathrm{CD} 8^{+}$cells was unaffected, the gene expression of CD8a was significantly lower in sponge treated muscles.

To gain further insight into the immune response within the first 7 days post-injury, we performed a PCR immunoarray. Of the 87 genes related to innate and adaptive immune 
responses, 35 genes in untreated group and 28 genes in treated group were significantly higher than the control group. The innate immune response is the first line of defense and is initiated by damage associated molecular patterns (DAMPs) such as cellular fragments or degraded ECM proteins that can stimulate TLRs and NLRs. The gene expression of TLRs-1, 5, 6 and 9 was significantly higher with sponge treatment compared to uninjured controls. TLRs have been implicated in the recognition of implanted biomaterials [27]. Besides antigen presenting cells of the innate immune system, the expression of TLRs has been reported on regenerating myofibers and vascular endothelial cells in inflammatory myopathies [35]. However, the localization of TLRs was not investigated in this study.

The complement system is a crucial component of innate immunity [37]. In this study, the gene expression complement opsonin C3 was significantly higher in the sponge treated muscles compared to uninjured controls. It has been shown that implantation of biomaterials can activate the complement system [38]. Besides initiating inflammation, the complement system also plays a role in muscle repair. For instance, muscle regeneration was found to be impaired following cardiotoxin injury in C3-deficient mice due to lower macrophage infiltration and decreased myoblast proliferation [39].

The implantation of FK-506 loaded sponges resulted in the downregulation of several inflammatory genes associated with T-cell activity such as CD8a, CXCL10, CXCR3, CD40, caspase 1, and FasL. Studies have shown that FK-506 can inhibit CXCL10 [40], CD8+ T-cell expansion, and FasL activity [41]. T-cells express CXCR3, a receptor for CXCL10. Binding of CXCL10 to CXCR3 initiates inflammation, promotes apoptosis, and reduces angiogenesis [40, 42-44]. CXCL10-CXCR3 axis is also important for peripheral homing of T-cells, and the gene expression of both these molecules was downregulated with sponge treatment on day 7 . T-cell activation requires CD40 ligand (CD40Lg) binding to CD40, which is expressed on antigenpresenting cells. It has been suggested that CD40-CD40Lg binding can directly and independently co-stimulate $\mathrm{T}$ cells [45]. The blockade of this interaction resulted in long-term cardiac allograft survival in a murine model, suggesting that inhibiting CD40 alone can suppress T-cell mediated immunogenic responses. The cytotoxic activity of CD8 cells is mediated by apoptosis-associated genes such as caspase 1 and FasL. Interestingly, FasL has been implicated in muscle atrophy and degeneration in Duchenne muscular dystrophy (DMD) patients [46]. 
Sponge treatment also resulted in significant upregulation of interferon- $\gamma($ IFN- $\gamma)$. IFN- $\gamma$ is primarily secreted by activated T-cells. It is an inflammatory cytokine that regulates various immune responses and physiological processes [47-49]. Inflammatory myopathies typically show increased type 1 interferon signaling and elevated expression of type I IFN stimulated genes (ISG) such as MX1, CXCL10, and IRF7 [50]. Atrophic small diameter myofibers in dermatomyositis patients have shown co-expression of atrogin 1 and MX1. A persistent IFNinduced response was implicated in the pathophysiology of the juvenile dermatomyositis, which is characterized by necrotic pathways and degeneration/regeneration cascades [51]. In sponge treated VML injured muscles, IFN- $\gamma$ was upregulated while ISGs such as MX2, CXCL10, and IRF7 were downregulated. The receptor, IFN- $\gamma$ R1, was also found downregulated in response to sponge treatment. These results suggest a potential negative regulation of IFN- $\gamma$ signaling by sponge treatment [52]. Several desensitization pathways for IFN- $\gamma$ signaling have been proposed [53]. However, more studies are needed to determine if ISGs exacerbate VML injury similar to inflammatory myopathies. While FK-506 has been effective against inflammatory myopathies in several studies $[54,55]$, it is unclear if it inhibits ISGs or IFN- $\gamma$ signaling in muscle injuries such as VML.

Taken together, FK-506 loaded sponges have an overall negative impact on T-cell associated gene expression within the first week post-injury, but this effect is short-lived. The peak in T-cell quantity on day 14 post-injury was almost synchronous with the clearance of FK506. Coinciding with an increased T-cell presence on day 14, an increase in the number of small diameter $\left(<500 \mu \mathrm{m}^{2}\right)$ myofibers, and MHC: COL ratio was also observed. T-cells play complex roles in skeletal muscle regeneration and show varied responses to muscular dystrophies and injuries. Pathological muscle conditions such as Duchenne Muscular Dystrophy or polymyositis are characterized by the increased and persistent presence of $\mathrm{CD}^{+} \mathrm{T}$-cells [25]. Studies have suggested that $\mathrm{CD} 8+\mathrm{T}$ cells could have a direct cytotoxic role on muscle fibers expressing major histocompatibility complex (MHC) class I molecules [56]. Healthy individuals do not show MHC class I myofibers, but they are frequently observed in myositis patients. In another study, depletion of $\mathrm{CD}^{+}$T-cells in cardiotoxin damaged Casitas B-lineage lymphoma-b (Cbl-b)deficient mice resulted in improved regenerative outcomes [26]. 
In contrast, other studies have indicated that T-cells might play a role in muscle regeneration, and completely abolishing T-cell response could have detrimental effects. For instance, in a model of cardiotoxin induced muscle injury, it was observed that in the absence of $\mathrm{CD}^{+} \mathrm{T}$-cells, matrix deposition is increased. At the same time, monocyte recruitment, myoblast proliferation, and myofiber growth are diminished [57]. In a study by Hurtgen et al., implantation of minced muscle autografts resulted in enhanced presence of $\mathrm{CD}^{+}, \mathrm{CD}^{+}$, and $\mathrm{CD}^{+} \mathrm{T}$ cells in a VML model [58]. The increased presence of these cell types over 14 days postinjury did not hinder muscle regeneration, as evidenced by newly regenerating myofibers and improved functional recovery. In a human study, increased presence of CD ${ }^{+} \mathrm{T}$-cells was implicated in muscle adaptation to repeated eccentric contractions $[59,60]$. The fact that $\mathrm{CD}^{+}$ T-cells were increased significantly only after second bout of exercise when evidence of muscle damage was reduced, suggested that these cells do not exacerbate injury but facilitate repair [59, 61]. Therefore, it appears that increased $\mathrm{CD}^{+} \mathrm{T}$-cell infiltration coincides with muscle repair, but the exact mechanism through which $\mathrm{CD}^{+} \mathrm{T}$-cells participate in muscle regeneration remains unknown and needs to be investigated in future studies.

Interestingly, we did not observe heightened myogenic gene or protein expression with sponge treatment of VML injury. However, quantitative analysis showed a significantly higher number of small diameter myofibers $\left(<500 \mu \mathrm{m}^{2}\right)$ with treatment. We believe that myofiber splitting could account for the significant increase in the small diameter myofibers on days 14 and 28 in the sponge treated muscles [62]. The leftward shift in the muscle fiber size distribution curve (Supplementary Figure 3) between days 14 and 28 suggests that larger myofibers may have split during this period. Qualitative analysis showed several myofibers with irregular shapes and displaced myonuclei, from which a smaller myofiber appeared to have broken apart (Supplementary Figure 3). Myofiber splitting has been observed in hypertrophy models [63], which indicates that the process may be an adaptation to maintain either the myonuclear domain, oxygen diffusion capacity, or force production [64]. In VML models, the remaining muscle mass experiences chronic overload as it attempts to compensate for the lost tissue [8]. It has been suggested that myofiber splitting in response to increased loading can be biomechanically advantageous as it distributes the force over a larger surface area [64]. Therefore, myofiber splitting may account for higher myofiber counts and peak isometric torque in sponge treated muscles. 
In this study, the sponge treated muscles showed several clusters of small-diameter myofibers, the majority of which appeared to be Type 1 or 2A myofibers. Quantitative analysis also confirmed significantly higher numbers of small diameter myofibers $\left(<500 \mu \mathrm{m}^{2}\right)$ in the fiber type 1 and 2A category. Taken together, these results might indicate that the slow oxidative fiber types are more prone to splitting in the VML model. We have also demonstrated that VML injury primarily impacts the CSA of fast glycolytic fibers (i.e., Type $2 \mathrm{~B}$ and $2 \mathrm{X}$ ). This result is in agreement with previous studies where eccentric contractions [65] and DMD [66] were shown to damage type 2 fibers selectively. Sponge treatment partially rescued the CSA of type $2 \mathrm{~B}$ but not $2 \mathrm{X}$ myofibers. Sponge treated muscles showed significantly higher CSA of type 2B myofibers as well as greater number of large diameter $\left(>4000 \mu \mathrm{m}^{2}\right)$ type $2 \mathrm{~B}$ fibers compared to untreated VML injured muscles. An improvement in type 2B myofiber CSA could also account for increased force production. These findings have major implications for VML injured patients because large type 2B fibers can withstand substantial loads. Therefore, preventing their loss and preserving their CSA might help improve the quality of life in affected patients [67].

Overall, these results suggest that implantation of biomimetic sponges protects the remaining muscle mass from necrosis and degeneration. Sponge treatment appears to reduce the severity of the injury and allows for improvements in muscle structure and function. These results could be attributed to FK-506 driven immunomodulation, replenishment of vital ECM proteins, and mechanical support offered by the three-dimensional scaffold. A potential limitation of this study is the rapid release of the unconjugated FK-506 that was encapsulated in the biomimetic sponges. FK-506 showed a rapid burst release in vitro as it diffused out of the sponges. We did not investigate the in vivo release kinetics of FK-506, but it can be reasonably assumed that the drug is cleared within the first few days after sponge implantation.

Interestingly, it has been shown that dendritic cells can sequester FK-506 and release it slowly in quantities that can inhibit T-cell activity for at least 72 hours [68]. Dendritic cells have been detected as early as 24 hours after muscle injury $[69,70]$. While continuous administration of FK-506 has been done in VML models [23, 24], others have reported that a single local application of FK-506 at the time of repair can support functional nerve regeneration 2-3 months after injury [71, 72]. Future studies may investigate the impact of sustained-release FK-506 on VML injury to determine if a short-term administration is adequate. However, in a clinical setting, prolonged immunomodulation can be easily achieved by administering FK-506 either as 
an oral dose or intravenous infusion [73]. Future work will also investigate the extent to which functional recovery can be enhanced by combining biomimetic sponge treatment with physical rehabilitation [74].

\section{Figure Captions:}

Figure 1. FK506 release from biomimetic sponges was measured in vitro. Regardless of initial concentration, most of the drug was released within the first three days. "*” indicates a statistical difference $(\mathrm{p}<0.05)$ in $25 \mu \mathrm{M}$ sponges versus $50 \mu \mathrm{M}$ and $100 \mu \mathrm{M}$ sponges at a particular timepoint.

Figure 2. Myogenic gene expression was determined by PCR. The quantification of (A) Pax7, (B) MyoD, (C) Myogenin, (D) eMHC, (F) TGF- $\beta 1$ and (G) COL1 fold expression is shown for days 7,14 , and 28 post-injury. “\#” indicates a statistical difference $(\mathrm{p}<0.05)$ from Day 7 and “ $\varphi$ ” indicates a statistical difference $(p<0.05)$ from Day 14 for a particular treatment group.

Figure 3. Flow cytometry was used to quantify the infiltrating T-cells. (A) The gating strategy is shown. The percentage of (B) total T-cells $\left(\mathrm{CD}^{+}\right)$, (C) helper T-cells (CD3 $\left.{ }^{+} \mathrm{CD}^{+}\right)$, and (D) cytotoxic T-cells $\left(\mathrm{CD}^{+} \mathrm{CD}^{+}\right)$cells were quantified. Injured muscles had higher percentages of T-cells compared to uninjured controls at day 7 post-injury. Treated muscles contained more Tcells than both untreated and uninjured muscles at day 14. By day 28, the T-cell response subsided. "*” indicates a statistical difference $(\mathrm{p}<0.05)$ between different treatment groups at a particular time-point. “\#” indicates a statistical difference $(p<0.05)$ from Day 7 and " $\varphi$ " indicates a statistical difference $(\mathrm{p}<0.05)$ from Day 14 for a particular treatment group.

Figure 4. Flow cytometry was used to quantify the infiltrating macrophages. (A) The gating strategy is shown. The percentage of $(\mathrm{B})$ total myeloid cells $\left(\mathrm{CD} 11 \mathrm{~b}^{+}\right),(\mathrm{C})$ pro-inflammatory cells $\left(\mathrm{CD} 11 \mathrm{~b}^{+} \mathrm{CD}^{+} 6^{+}\right)$, and (D) anti-inflammatory $\left(\mathrm{CD} 11 \mathrm{~b}^{+} \mathrm{CD} 163^{+}\right)$cells were quantified. At days 7 and 14 post-injury, total myeloid cells and pro-inflammatory cells were elevated in injured muscles. Anti-inflammatory $\left(\mathrm{CD} 11 \mathrm{~b}^{+} \mathrm{CD} 163^{+}\right)$cells were increased in injured muscles at day 7 , but only remained elevated in treated muscles at day 14. The myeloid cell response subsided in injured muscles by day 28. “*” indicates a statistical difference $(\mathrm{p}<0.05)$ between different treatment groups at a particular time-point. “\#” indicates a statistical difference ( $<<$ 
$0.05)$ from Day 7 and “ $\varphi$ " indicates a statistical difference $(p<0.05)$ from Day 14 for a particular treatment group.

Figure 5. An array of immunogenic markers was quantified via PCR. (A) The table shows specific genes and their fold changes in both untreated and treated muscles at 7 days post-injury. “*” indicates statistical differences $(p<0.05)$ between injured muscles and controls. “ $\uparrow "$ indicates statistical differences $(\mathrm{p}<0.05)$ between untreated and treated muscles. (B) Genes downregulated and upregulated with sponge-treatment are shown. (C) A schematic of the implicated immunological events is presented.

Figure 6. (A) Tibialis anterior muscles were weighed upon collection at days 7, 14, and 28 postinjury. (B) Peak isometric torque was measured and (C) normalized to body weight and (D) TA weight. (E) Qualitative analysis of injured muscles showed no differences in the prevalence of post-synaptic terminals. "*” indicates a statistical difference $(\mathrm{p}<0.05)$ between different treatment groups at a particular time-point. “\#” indicates a statistical difference $(p<0.05)$ from Day 7 for a particular treatment group.

Figure 7. Transverse muscle cross-sections were stained with H\&E and whole slide images were captured at days 7, 14, and 28 post-injury. Treated muscles had more myofibers with centrally located nuclei at day 28. “*” indicates a statistical difference $(p<0.05)$ between different treatment groups. The black arrow indicates the sponge in the defect.

Figure 8. Transverse muscle cross-sections were stained with myosin heavy chain (MHC), collagen (COL), and DAPI. The \% area of MHC and COL were quantified and MHC:COL ratios were calculated. Treated muscles have higher MHC:COL ratios by day 28 post-injury. “*” indicates a statistical difference $(\mathrm{p}<0.05)$ between different treatment groups at a particular time-point. The white arrow indicates the sponge in the defect.

Figure 9. Muscles were stained for (A) T-cells (CD3), (B) macrophages (CD68), (C) endothelial cells (CD31), and (D) stem cells (SCA-1). The white asterisk indicates the sponge in the defect.

Figure 10. Muscle fibers were outlined with laminin and cross-sectional areas were quantified. Myofiber CSA at day 28 for (A) cage control, (B) untreated, and (C) treated groups are displayed as color-coded maps. (D) A higher quantity of small CSA fibers $\left(<500 \mu \mathrm{m}^{2}\right)$ were present in 
treated muscles at both days 14 and 28 post-injury. (E) Treated muscles also contained higher numbers of total myofibers than untreated muscles at days 14 and 28. (F) The mean CSA was not different between untreated and treated groups. "*” indicates a statistical difference $(p<0.05)$ between untreated and treated muscles at a particular time-point. " $\gamma$ " indicates a statistical difference $(p<0.05)$ between controls and injured muscles at a particular time-point. " $\varphi$ " indicates a statistical difference $(\mathrm{p}<0.05)$ between day 14 and 28 for a particular treatment group. The dashed line indicates control.

Figure 11. Muscle cross-sections were stained for different fast- and slow- twitch fiber types. Immunostained images and color-coded maps are displayed for (A) uninjured controls, (B) untreated muscles, and (C) sponge-treated muscles. Fiber type distribution and CSA was quantified. (D) VML had no effect on the percentages of different types of fibers, but (E) decreased the mean CSA of fast-twitch fibers (i.e., type 2B and 2X). Sponge treatment increased the mean CSA of Type 2B fibers compared to untreated muscles. Fiber type CSA distributions for (F) Type 1, (G) Type 2A, (H) Type 2B, and (I) Type 2X myofibers are shown. “*” indicates a statistical difference $(\mathrm{p}<0.05)$ between different treatment groups.

Supplemental Figure 1. Full-scale images of muscle sections stained with MHC and COL were used to quantify the percent area of each. Thresholding without separating the remaining muscle tissue and the defect region (top) results in a less representative collagen \% area. By contrast, splitting the remaining muscle tissue and defect region into two separate images (bottom) results in higher accuracy because the defect can be detected without muscle fibers being filled in with collagen.

Supplemental Figure 2. Higher magnification images provide a closer look at the defect region. Sponge treated muscles show more small-diameter myosin ${ }^{+}$fibers at day 14 post-injury.

Supplemental Figure 3. Qualitative analysis of split myofibers is presented. Arrows show the branched/split myofibers. Muscle cross-sectional areas are represented as a fiber size distribution curve. "*” indicates a statistical difference $(p<0.05)$ between different groups.

Acknowledgments: This work was supported by a grant from the National Institute of Health (NIGMS) 1R15GM129731 awarded to KG. We would like to thank Joy Eslick and Sherri Koehm for help with the flow cytometry experiments. We would like to thank Gary D. London 
(Washington University), as well as Caroline Murphy and Dr. Grant Kolar (Saint Louis University) for technical assistance with histological imaging.

Conflict of Interest: KG has equity interest in GenAssist, Inc., and serves on the company's scientific advisory board. GenAssist, Inc. is developing products related to the research described in this paper. The terms of this arrangement have been reviewed and approved by Saint Louis University, in accordance with its conflict of interest policies. GJH is the CEO and JM is the CTO of GenAssist, Inc. and both are members of the board of directors. AJD also holds equity interest in GenAssist, Inc.

\section{References}

1. Garg, K., C.L. Ward, C.R. Rathbone, et al., Transplantation of devitalized muscle scaffolds is insufficient for appreciable de novo muscle fiber regeneration after volumetric muscle loss injury. Cell Tissue Res, 2014. 358(3): p. 857-73.

2. Garg, K., C.L. Ward, B.J. Hurtgen, et al., Volumetric muscle loss: persistent functional deficits beyond frank loss of tissue. J Orthop Res, 2015. 33(1): p. 40-6.

3. Garg, K., B.T. Corona, and T.J. Walters, Losartan administration reduces fibrosis but hinders functional recovery after volumetric muscle loss injury. J Appl Physiol (1985), 2014. 117(10): p. 1120-31.

4. Greising, S.M., J.C. Rivera, S.M. Goldman, et al., Unwavering Pathobiology of Volumetric Muscle Loss Injury. Sci Rep, 2017. 7(1): p. 13179.

5. Corona, B.T., K. Garg, C.L. Ward, et al., Autologous minced muscle grafts: a tissue engineering therapy for the volumetric loss of skeletal muscle. Am J Physiol Cell Physiol, 2013. 305(7): p. C761-75.

6. Bianchi, B., C. Copelli, S. Ferrari, et al., Free flaps: outcomes and complications in head and neck reconstructions. J Craniomaxillofac Surg, 2009. 37(8): p. 438-42.

7. Urken, M.L., H. Weinberg, D. Buchbinder, et al., Microvascular free flaps in head and neck reconstruction. Report of 200 cases and review of complications. Arch Otolaryngol Head Neck Surg, 1994. 120(6): p. 633-40.

8. Aurora, A., K. Garg, B.T. Corona, et al., Physical rehabilitation improves muscle function following volumetric muscle loss injury. BMC Sports Sci Med Rehabil, 2014. 6(1): p. 41.

9. Garg, K. and M.D. Boppart, Influence of exercise and aging on extracellular matrix composition in the skeletal muscle stem cell niche. J Appl Physiol (1985), 2016. 121(5): p. 1053-1058.

10. Dunn, A., M. Talovic, K. Patel, et al., Biomaterial and stem cell-based strategies for skeletal muscle regeneration. J Orthop Res, 2019. 37(6): p. 1246-1262.

11. Larouche, J., S.M. Greising, B.T. Corona, et al., Robust inflammatory and fibrotic signaling following volumetric muscle loss: a barrier to muscle regeneration. Cell Death Dis, 2018. 9(3): p. 409.

12. Garg, K., B.T. Corona, and T.J. Walters, Therapeutic strategies for preventing skeletal muscle fibrosis after injury. Front Pharmacol, 2015. 6: p. 87.

13. Aguilar, C.A., S.M. Greising, A. Watts, et al., Multiscale analysis of a regenerative therapy for treatment of volumetric muscle loss injury. Cell Death Discov, 2018. 4: p. 33. 
14. Garg, K., C.L. Ward, and B.T. Corona, Asynchronous inflammation and myogenic cell migration limit muscle tissue regeneration mediated by a cellular scaffolds. Inflamm Cell Signal, 2014. 1(4).

15. Aurora, A., J.L. Roe, B.T. Corona, et al., An acellular biologic scaffold does not regenerate appreciable de novo muscle tissue in rat models of volumetric muscle loss injury. Biomaterials, 2015. 67: p. 393-407.

16. Aurora, A., B.T. Corona, and T.J. Walters, A Porcine Urinary Bladder Matrix Does Not Recapitulate the Spatiotemporal Macrophage Response of Muscle Regeneration after Volumetric Muscle Loss Injury. Cells Tissues Organs, 2016. 202(3-4): p. 189-201.

17. Hurtgen, B.J., C.L. Ward, K. Garg, et al., Severe muscle trauma triggers heightened and prolonged local musculoskeletal inflammation and impairs adjacent tibia fracture healing. J Musculoskelet Neuronal Interact, 2016. 16(2): p. 122-34.

18. Dumont, F.J., FK506, an immunosuppressant targeting calcineurin function. Curr Med Chem, 2000. 7(7): p. 731-48.

19. Szabo, G., C. Gavala, and P. Mandrekar, Tacrolimus and cyclosporine A inhibit allostimulatory capacity and cytokine production of human myeloid dendritic cells. J Investig Med, 2001. 49(5): p. 442-9.

20. Jennings, C., B. Kusler, and P.P. Jones, Calcineurin inactivation leads to decreased responsiveness to LPS in macrophages and dendritic cells and protects against LPS-induced toxicity in vivo. Innate Immun, 2009. 15(2): p. 109-20.

21. Thomson, D.M. and S.E. Gordon, Diminished overload-induced hypertrophy in aged fast-twitch skeletal muscle is associated with AMPK hyperphosphorylation. J Appl Physiol (1985), 2005. 98(2): p. 557-64.

22. Arias-Diaz, J., J.A. Ildefonso, J.J. Munoz, et al., Both tacrolimus and sirolimus decrease Th1/Th2 ratio, and increase regulatory $T$ lymphocytes in the liver after ischemia/reperfusion. Lab Invest, 2009. 89(4): p. 433-45.

23. Hurtgen, B.J., B.E.P. Henderson, C.L. Ward, et al., Impairment of early fracture healing by skeletal muscle trauma is restored by FK506. BMC musculoskeletal disorders, 2017. 18(1): p. 253-253.

24. Corona, B.T., J.C. Rivera, J.C. Wenke, et al., Tacrolimus as an adjunct to autologous minced muscle grafts for the repair of a volumetric muscle loss injury. J Exp Orthop, 2017. 4(1): p. 36.

25. Haas, G.J., A.J. Dunn, M. Marcinczyk, et al., Biomimetic sponges for regeneration of skeletal muscle following trauma. J Biomed Mater Res A, 2019. 107(1): p. 92-103.

26. Goodman, C.A., J.A. Kotecki, B.L. Jacobs, et al., Muscle fiber type-dependent differences in the regulation of protein synthesis. PLoS One, 2012. 7(5): p. e37890.

27. Shokouhi, B., C. Coban, V. Hasirci, et al., The role of multiple toll-like receptor signalling cascades on interactions between biomedical polymers and dendritic cells. Biomaterials, 2010. 31(22): p. 5759-71.

28. Chen, H.C., B. Sun, K.K. Tran, et al., Effects of particle size on toll-like receptor 9-mediated cytokine profiles. Biomaterials, 2011. 32(6): p. 1731-7.

29. Corona, B.T., X. Wu, C.L. Ward, et al., The promotion of a functional fibrosis in skeletal muscle with volumetric muscle loss injury following the transplantation of muscle-ECM. Biomaterials, 2013. 34(13): p. 3324-35.

30. Kasukonis, B., J. Kim, L. Brown, et al., Codelivery of Infusion Decellularized Skeletal Muscle with Minced Muscle Autografts Improved Recovery from Volumetric Muscle Loss Injury in a Rat Model. Tissue Eng Part A, 2016. 22(19-20): p. 1151-1163.

31. Passipieri, J.A., H.B. Baker, M. Siriwardane, et al., Keratin Hydrogel Enhances In Vivo Skeletal Muscle Function in a Rat Model of Volumetric Muscle Loss. Tissue Eng Part A, 2017. 23(11-12): p. 556-571. 
32. Sicari, B.M., J.P. Rubin, C.L. Dearth, et al., An acellular biologic scaffold promotes skeletal muscle formation in mice and humans with volumetric muscle loss. Science translational medicine, 2014. 6(234): p. 234ra58-234ra58.

33. Corona, B.T., M.A. Machingal, T. Criswell, et al., Further development of a tissue engineered muscle repair construct in vitro for enhanced functional recovery following implantation in vivo in a murine model of volumetric muscle loss injury. Tissue Eng Part A, 2012. 18(11-12): p. 121328.

34. Corona, B.T., C.L. Ward, H.B. Baker, et al., Implantation of in vitro tissue engineered muscle repair constructs and bladder acellular matrices partially restore in vivo skeletal muscle function in a rat model of volumetric muscle loss injury. Tissue Engineering Part A, 2013. 20(3-4): p. 705715.

35. Quarta, M., M. Cromie, R. Chacon, et al., Bioengineered constructs combined with exercise enhance stem cell-mediated treatment of volumetric muscle loss. Nat Commun, 2017. 8: p. 15613.

36. Fishman, J.M., M.W. Lowdell, L. Urbani, et al., Immunomodulatory effect of a decellularized skeletal muscle scaffold in a discordant xenotransplantation model. Proc Natl Acad Sci U S A, 2013. 110(35): p. 14360-5.

37. Zhang, L., Y. Dong, Y. Dong, et al., Role of integrin-beta 3 protein in macrophage polarization and regeneration of injured muscle. J Biol Chem, 2012. 287(9): p. 6177-86.

38. Modinger, Y., G.Q. Teixeira, C. Neidlinger-Wilke, et al., Role of the Complement System in the Response to Orthopedic Biomaterials. Int J Mol Sci, 2018. 19(11).

39. Zhang, C., C. Wang, Y. Li, et al., Complement C3a signaling facilitates skeletal muscle regeneration by regulating monocyte function and trafficking. Nat Commun, 2017. 8(1): p. 2078.

40. Tiefenthaler, M., S. Hofer, S. Ebner, et al., In vitro treatment of dendritic cells with tacrolimus: impaired T-cell activation and IP-10 expression. Nephrol Dial Transplant, 2004. 19(3): p. 553-60.

41. Imado, T., T. Iwasaki, T. Kuroiwa, et al., Effect of FK506 on donor T-cell functions that are responsible for graft-versus-host disease and graft-versus-leukemia effect. Transplantation, 2004. 77(3): p. 391-8.

42. Crescioli, C., M. Sottili, P. Bonini, et al., Inflammatory response in human skeletal muscle cells: CXCL10 as a potential therapeutic target. Eur J Cell Biol, 2012. 91(2): p. 139-49.

43. Ishiuchi, Y., H. Sato, K. Tsujimura, et al., Skeletal muscle cell contraction reduces a novel myokine, chemokine (C-X-C motif) ligand 10 (CXCL10): potential roles in exercise-regulated angiogenesis. Biosci Biotechnol Biochem, 2018. 82(1): p. 97-105.

44. Kim, J., J.Y. Choi, S.H. Park, et al., Therapeutic effect of anti-C-X-C motif chemokine 10 (CXCL10) antibody on C protein-induced myositis mouse. Arthritis Res Ther, 2014. 16(3): p. R126.

45. Hancock, W.W., M.H. Sayegh, X.G. Zheng, et al., Costimulatory function and expression of CD40 ligand, CD80, and CD86 in vascularized murine cardiac allograft rejection. Proc Natl Acad Sci U S A, 1996. 93(24): p. 13967-72.

46. Abdel-Salam, E., I. Abdel-Meguid, and S.S. Korraa, Markers of degeneration and regeneration in Duchenne muscular dystrophy. Acta Myol, 2009. 28(3): p. 94-100.

47. Cheng, M., M.H. Nguyen, G. Fantuzzi, et al., Endogenous interferon-gamma is required for efficient skeletal muscle regeneration. Am J Physiol Cell Physiol, 2008. 294(5): p. C1183-91.

48. Foster, W., Y. Li, A. Usas, et al., Gamma interferon as an antifibrosis agent in skeletal muscle. J Orthop Res, 2003. 21(5): p. 798-804.

49. Londhe, P. and J.K. Davie, Gamma interferon modulates myogenesis through the major histocompatibility complex class II transactivator, CIITA. Mol Cell Biol, 2011. 31(14): p. 2854-66.

50. Cappelletti, C., F. Baggi, F. Zolezzi, et al., Type I interferon and Toll-like receptor expression characterizes inflammatory myopathies. Neurology, 2011. 76(24): p. 2079-88. 
51. Tezak, Z., E.P. Hoffman, J.L. Lutz, et al., Gene expression profiling in DQA1*0501+ children with untreated dermatomyositis: a novel model of pathogenesis. J Immunol, 2002. 168(8): p. 415463.

52. Ladislau, L., X. Suarez-Calvet, S. Toquet, et al., JAK inhibitor improves type I interferon induced damage: proof of concept in dermatomyositis. Brain, 2018. 141(6): p. 1609-1621.

53. Schneider, W.M., M.D. Chevillotte, and C.M. Rice, Interferon-stimulated genes: a complex web of host defenses. Annu Rev Immunol, 2014. 32: p. 513-45.

54. Matsubara, S., K. Kondo, K. Sugaya, et al., Effects of tacrolimus on dermatomyositis and polymyositis: a prospective, open, non-randomized study of nine patients and a review of the literature. Clin Rheumatol, 2012. 31(10): p. 1493-8.

55. Ge, Y., H. Zhou, J. Shi, et al., The efficacy of tacrolimus in patients with refractory dermatomyositis/polymyositis: a systematic review. Clin Rheumatol, 2015. 34(12): p. 2097-103.

56. Venalis, P. and I.E. Lundberg, Immune mechanisms in polymyositis and dermatomyositis and potential targets for therapy. Rheumatology (Oxford), 2014. 53(3): p. 397-405.

57. Zhang, J., Z. Xiao, C. Qu, et al., CD8 T cells are involved in skeletal muscle regeneration through facilitating MCP-1 secretion and Gr1(high) macrophage infiltration. J Immunol, 2014. 193(10): p. 5149-60.

58. Hurtgen, B.J., C.L. Ward, C.M. Leopold Wager, et al., Autologous minced muscle grafts improve endogenous fracture healing and muscle strength after musculoskeletal trauma. Physiol Rep, 2017. 5(14).

59. Hyldahl, R.D., T.C. Chen, and K. Nosaka, Mechanisms and Mediators of the Skeletal Muscle Repeated Bout Effect. Exerc Sport Sci Rev, 2017. 45(1): p. 24-33.

60. Deyhle, M.R., A.M. Gier, K.C. Evans, et al., Skeletal Muscle Inflammation Following Repeated Bouts of Lengthening Contractions in Humans. Front Physiol, 2015. 6: p. 424.

61. Deyhle, M.R. and R.D. Hyldahl, The Role of T Lymphocytes in Skeletal Muscle Repair From Traumatic and Contraction-Induced Injury. Front Physiol, 2018. 9: p. 768.

62. Murach, K.A., S.H. White, Y. Wen, et al., Differential requirement for satellite cells during overload-induced muscle hypertrophy in growing versus mature mice. Skelet Muscle, 2017. 7(1): p. 14.

63. Soffe, Z., H.G. Radley-Crabb, C. McMahon, et al., Effects of loaded voluntary wheel exercise on performance and muscle hypertrophy in young and old male C57BI/6J mice. Scand J Med Sci Sports, 2016. 26(2): p. 172-88.

64. Murach, K.A., C.M. Dungan, C.A. Peterson, et al., Muscle Fiber Splitting Is a Physiological Response to Extreme Loading in Animals. Exerc Sport Sci Rev, 2019. 47(2): p. 108-115.

65. Lieber, R.L. and J. Friden, Selective damage of fast glycolytic muscle fibres with eccentric contraction of the rabbit tibialis anterior. Acta Physiol Scand, 1988. 133(4): p. 587-8.

66. Webster, C., L. Silberstein, A.P. Hays, et al., Fast muscle fibers are preferentially affected in Duchenne muscular dystrophy. Cell, 1988. 52(4): p. 503-13.

67. Gehrig, S.M., R. Koopman, T. Naim, et al., Making fast-twitch dystrophic muscles bigger protects them from contraction injury and attenuates the dystrophic pathology. Am J Pathol, 2010. 176(1): p. 29-33.

68. Orange, D.E., N.E. Blachere, J. Fak, et al., Dendritic cells loaded with FK506 kill T cells in an antigen-specific manner and prevent autoimmunity in vivo. Elife, 2013. 2: p. e00105.

69. Pimorady-Esfahani, A., M.D. Grounds, and P.G. McMenamin, Macrophages and dendritic cells in normal and regenerating murine skeletal muscle. Muscle Nerve, 1997. 20(2): p. 158-66.

70. Wirsdorfer, F., J.M. Bangen, E. Pastille, et al., Dendritic Cell-Like Cells Accumulate in Regenerating Murine Skeletal Muscle after Injury and Boost Adaptive Immune Responses Only upon a Microbial Challenge. PLoS One, 2016. 11(5): p. e0155870. 
71. Azizi, S., R. Mohammadi, K. Amini, et al., Effects of topically administered FK506 on sciatic nerve regeneration and reinnervation after vein graft repair of short nerve gaps. Neurosurg Focus, 2012. 32(5): p. E5.

72. Diaz, L.M., M.H. Steele, A.B. Guerra, et al., The role of topically administered FK506 (tacrolimus) at the time of facial nerve repair using entubulation neurorrhaphy in a rabbit model. Ann Plast Surg, 2004. 52(4): p. 407-13.

73. Venkataramanan, R., A. Jain, V.S. Warty, et al., Pharmacokinetics of FK 506 in transplant patients. Transplant Proc, 1991. 23(6): p. 2736-40.

74. Greising, S.M., C.L. Dearth, and B.T. Corona, Regenerative and Rehabilitative Medicine: A Necessary Synergy for Functional Recovery from Volumetric Muscle Loss Injury. Cells Tissues Organs, 2016. 202(3-4): p. 237-249. 


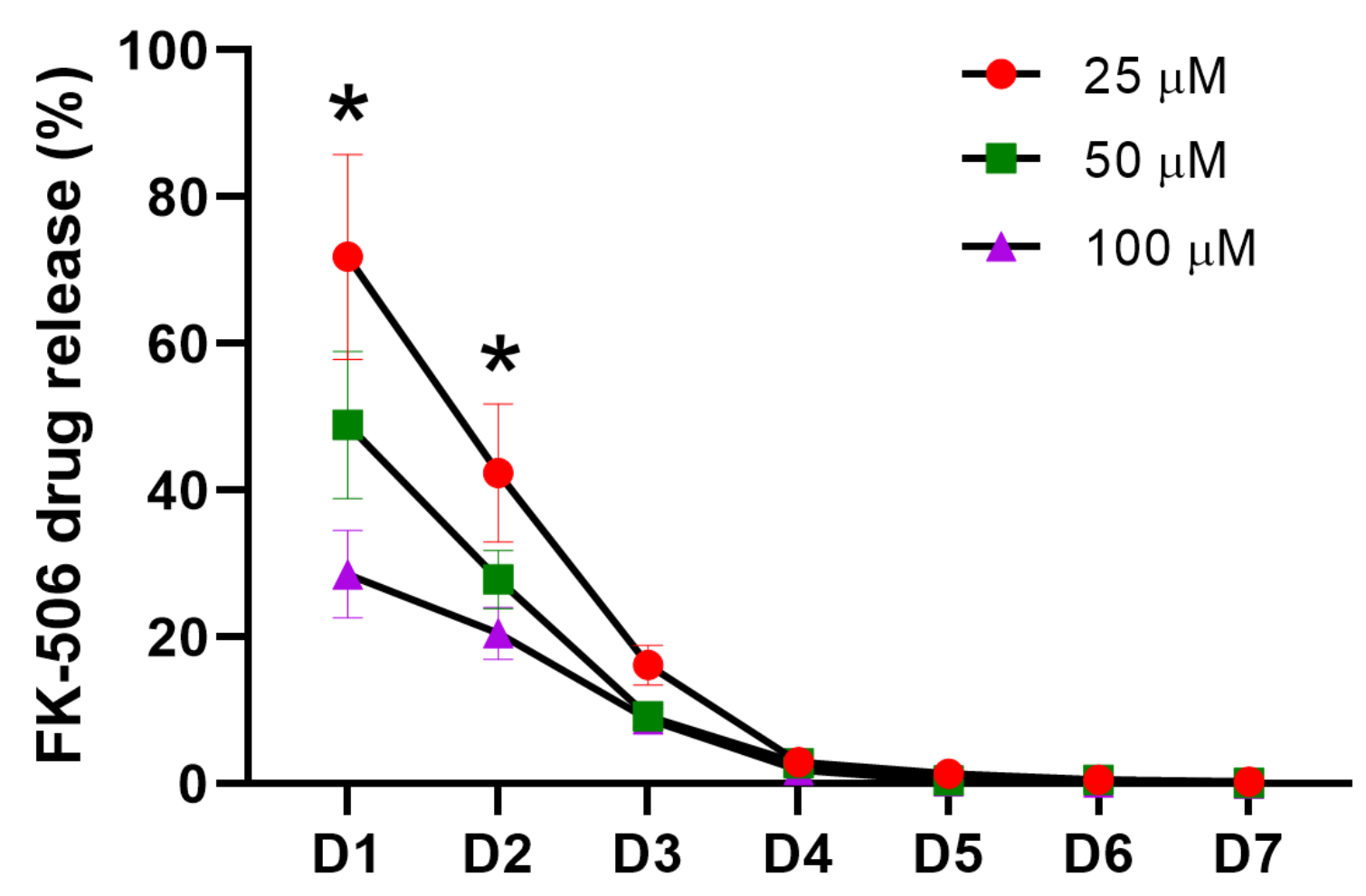

Fig. 1 

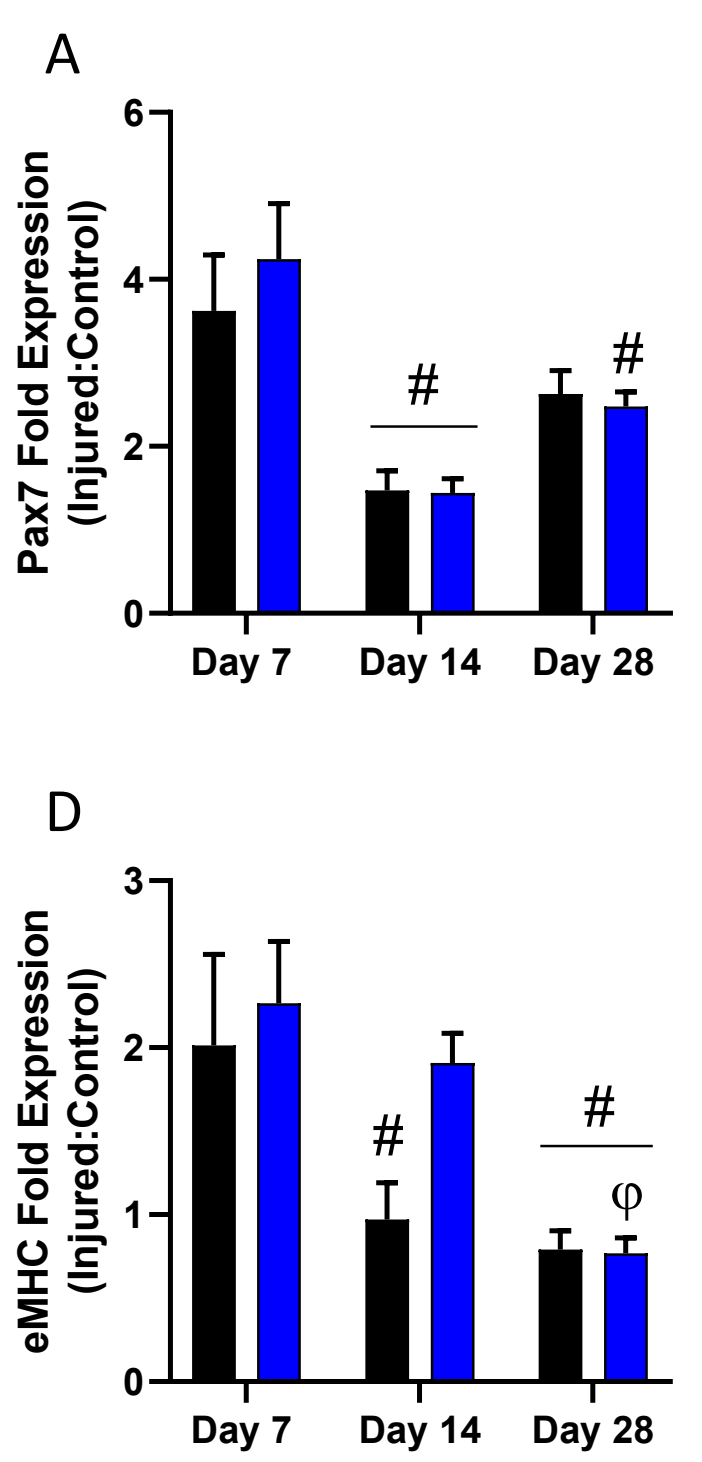

B

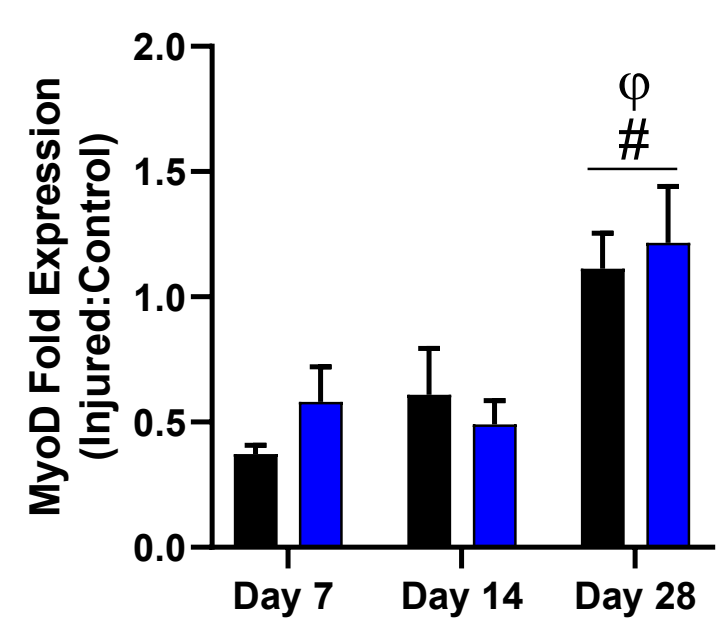

E

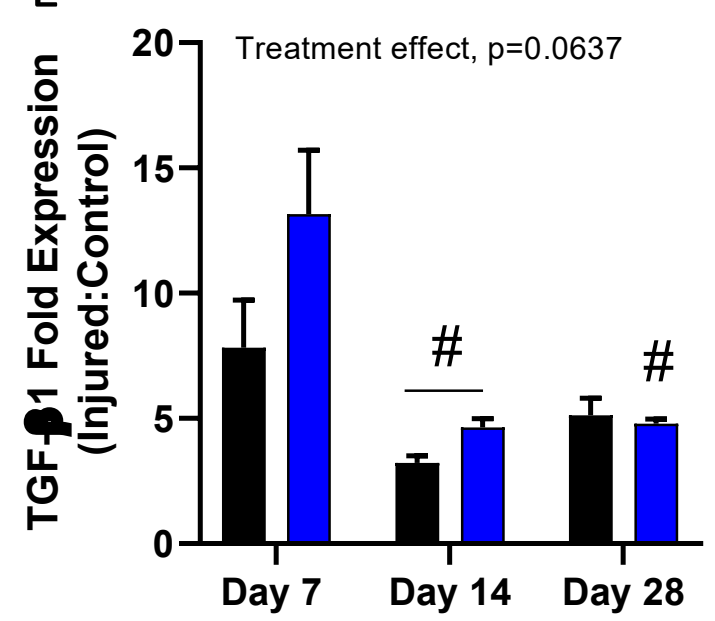

C

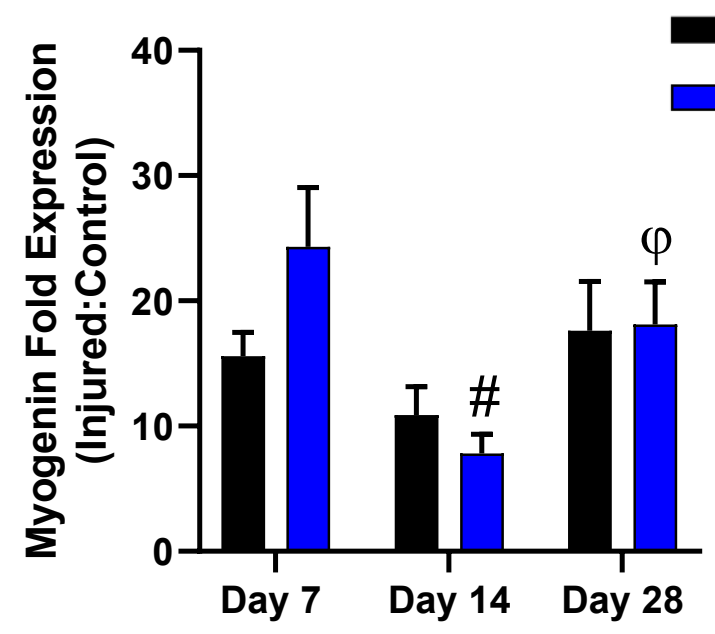

F

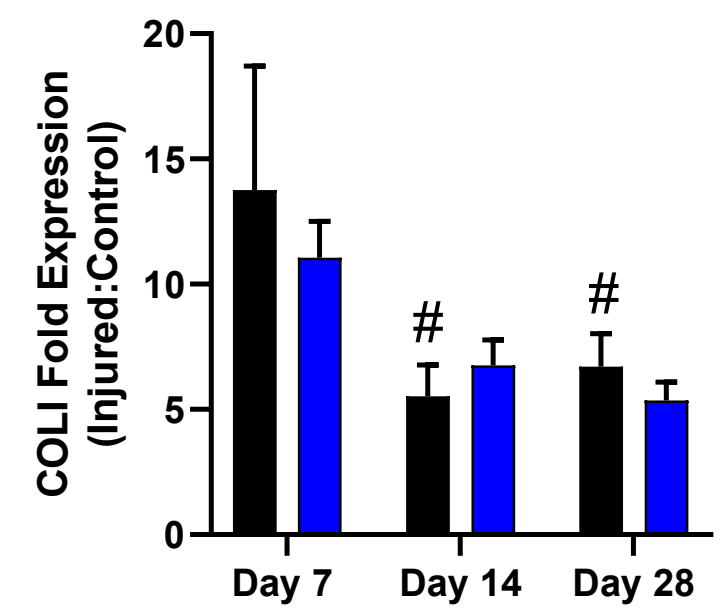

Fig. 2 


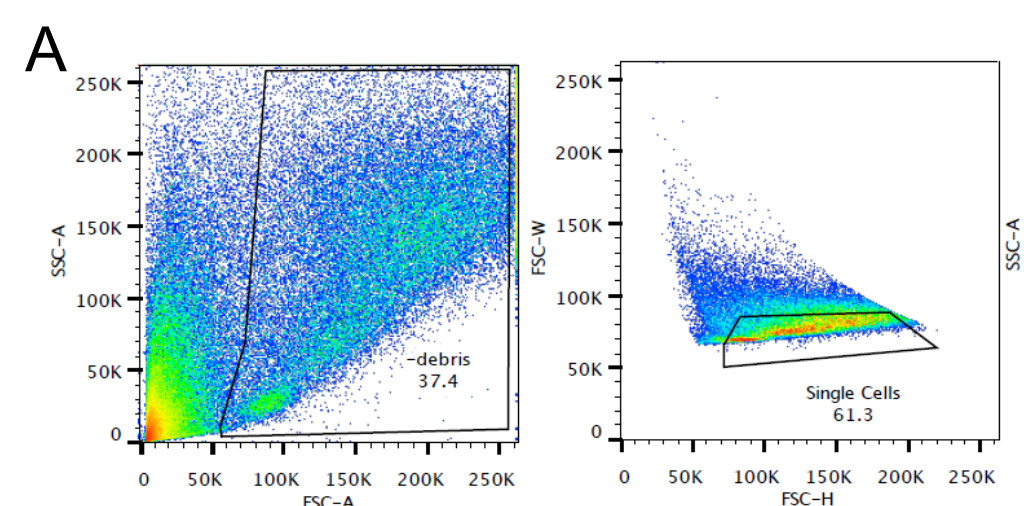

Exclude debris $\rightarrow$ Exclude doublets

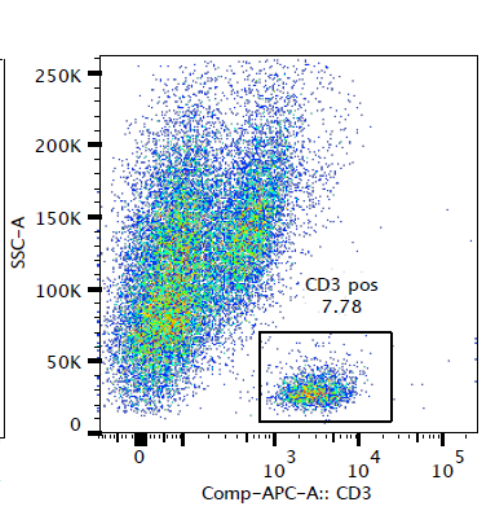

CD3+

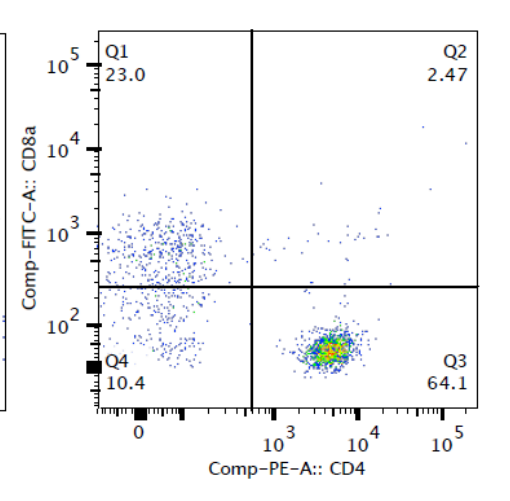

T Lymphocytes

D

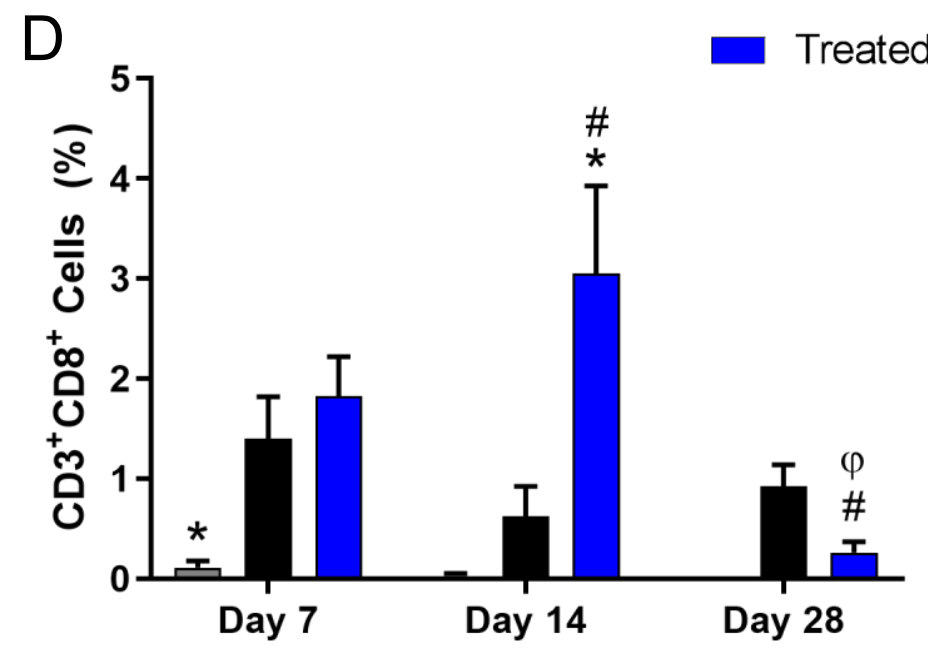

$\square$ Control

- Untreated
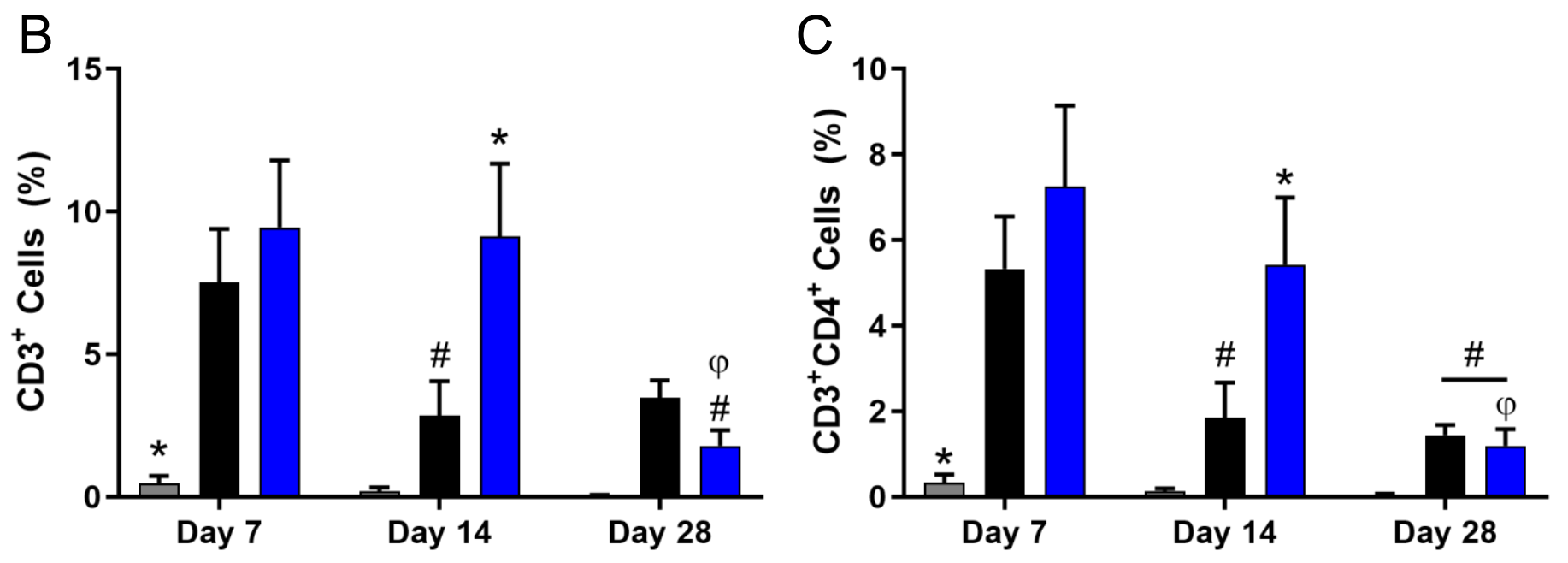

\section{Fig. 3}



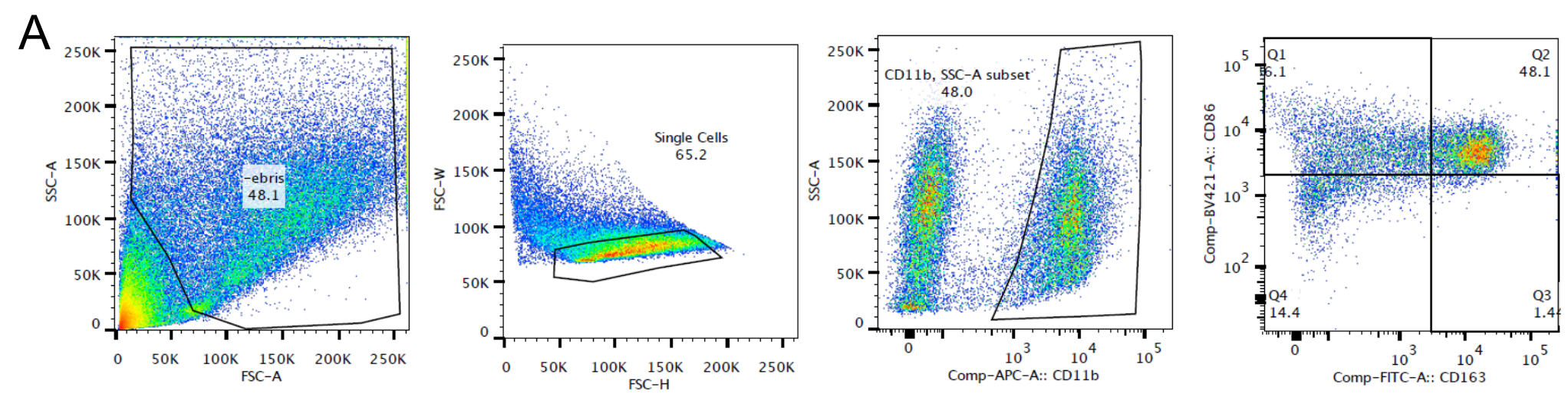

Exclude debris $\rightarrow$ Exclude doublets CD11b+

Macrophages

$\square$ Control

- Untreated
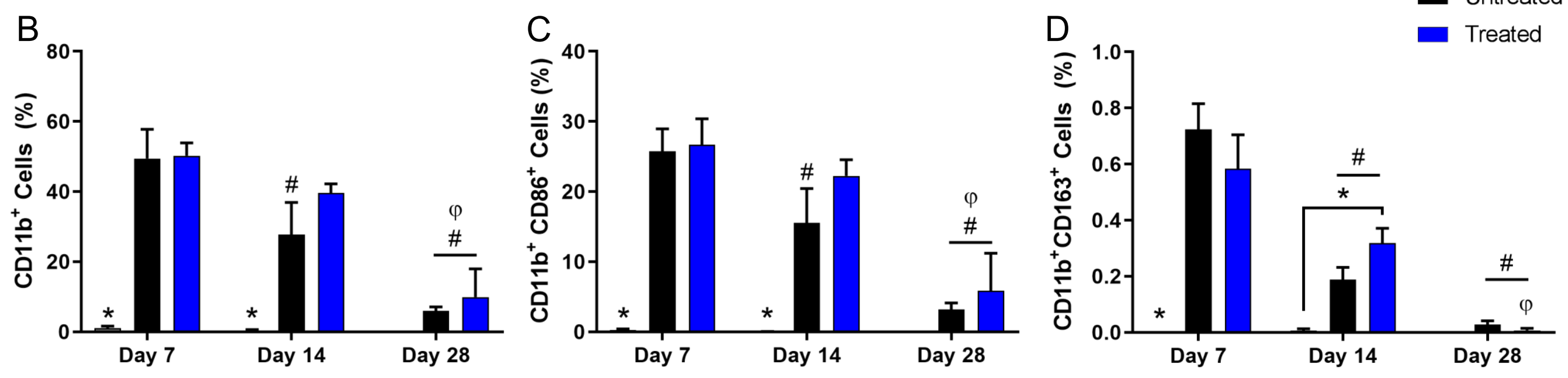

Fig. 4 


\section{A}
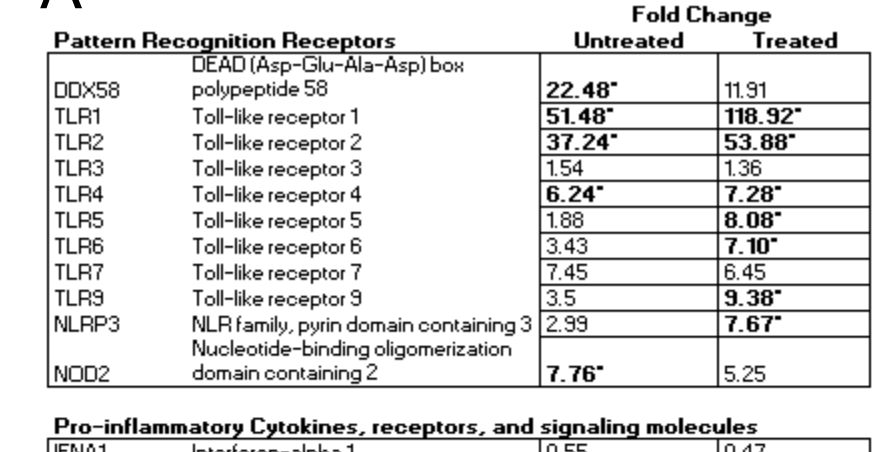

\begin{tabular}{|c|c|c|c|}
\hline IFNA1 & Interferon-alpha 1 & 0.55 & 0.47 \\
\hline IENAE1 1 - & Interferon (alpha, beta and omega) & & \\
\hline IFNE1 & Interferon beta 1 , fibroblast & $\frac{2.3}{2175}$ & 4.04 \\
\hline IFNGR1 & Interferon & 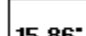 & $\log 1$ \\
\hline & Interferon gamma receptor 1 & & \\
\hline IL18 & Interleukin 18 & $24.35^{-}$ & $45.42^{-}$ \\
\hline ILAAA & Interleukin 1 alpha & 10.04 & \\
\hline Lie & Interleukin 1beta & $21.19^{-}$ & $21.38^{-}$ \\
\hline ILIR1 & Interleukin 1receptor, type I & $\frac{14.60^{-}}{0.32}$ & $6.86^{-1}$ \\
\hline IL23A & Interleukin 23, alpha subunit p19 & 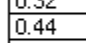 & \\
\hline IL5 & Interleukin 5 & & \\
\hline & Interleukin 6 & $57.84^{-}$ & $85.33^{7}$ \\
\hline & $\begin{array}{l}\text { Tumor necrosis factor } \\
\text { (TMFF superfanily member 2] }\end{array}$ & 2.93 & 251 \\
\hline & Signal transducer and activator of & $5.41^{\circ}$ & 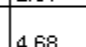 \\
\hline & $\begin{array}{l}\text { transoription } 1 \\
\text { Signal transducer and activator of }\end{array}$ & & \\
\hline АТЗ & 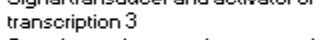 & $20.26^{\prime}$ & $30.03^{-}$ \\
\hline & $\begin{array}{l}\text { Signal trangsducer and activator of } \\
\text { transsirtion } 4\end{array}$ & $12.09^{-}$ & \\
\hline RORC & RAR-related orphan receptor $\mathrm{C}$ & $0.11^{-}$ & $0.06^{-1}$ \\
\hline CD14 & CD14 molecule & & 4.58 \\
\hline $\begin{array}{l}\text { CDIT1 } \\
\text { JAKK2 }\end{array}$ & $\begin{array}{l}\text { CDIf1molecule } \\
\text { Janus kinase } 2\end{array}$ & $\frac{0.12}{9.52^{-}}$ & $\mid \begin{array}{l}0.08 \\
9.35^{*}\end{array}$ \\
\hline
\end{tabular}

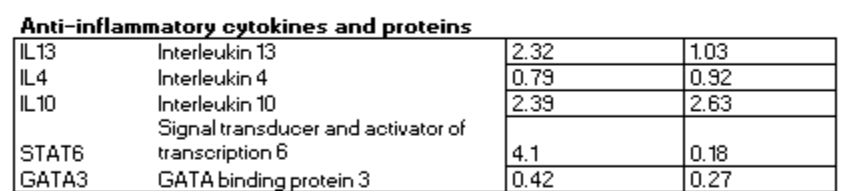

\begin{tabular}{ll} 
STAT6 & transcription 6 \\
GATA3 & GATA binding protein 3 \\
\hline
\end{tabular}

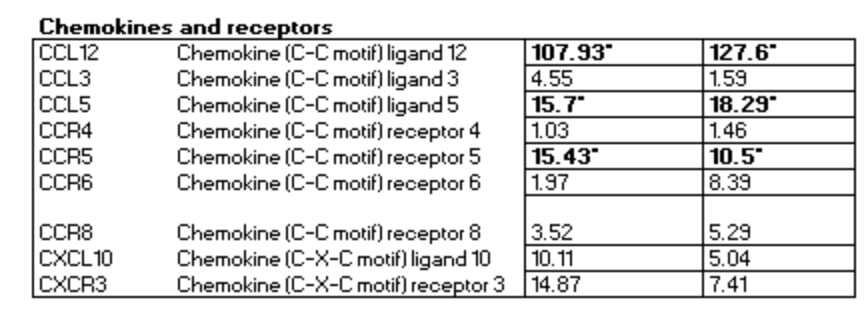

Fig. 5
B

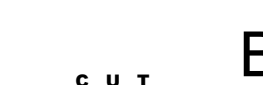

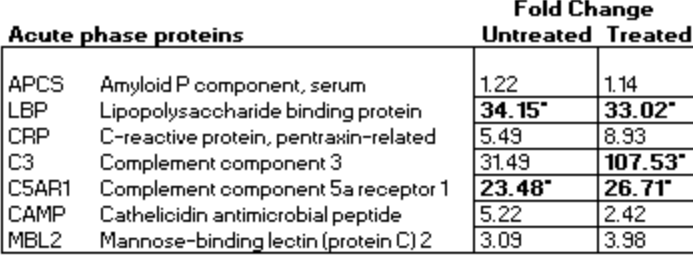

\section{Cell adhesion proteins
ITGAM Integrin, alphaM}

\begin{tabular}{|l|l|l|}
\hline ICAMM Integrin, alpha M & $21.51^{\circ}$ & 21.88 \\
\cline { 2 - 4 } & $\mathbf{1 6 . 7 1 ^ { - }}$ & $\mathbf{1 4 . 2 0 ^ { - }}$ \\
\hline
\end{tabular} Enzymes
\begin{tabular}{|ll|l|l|}
\hline LYZ2 & Lysozyme 2 & $\mathbf{3 6 . 0 8 ^ { * }}$ & $\mathbf{3 4 . 3 ^ { - }}$ \\
\cline { 2 - 4 } MPO & Myeloperoxidase & 3.21 & 5.03 \\
\hline
\end{tabular} NFkB signaling pathr ay molecules

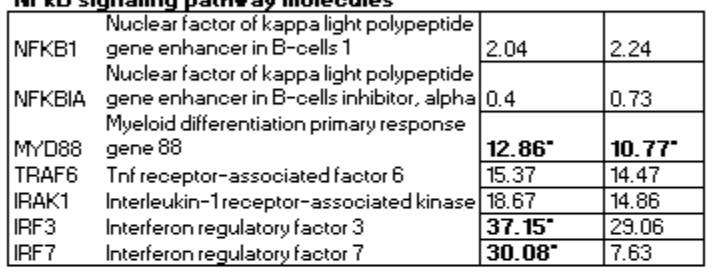

\section{Cell Survival and grovth}

\begin{tabular}{|ll|l|l|}
\hline MAPK1 Mitogen activated protein kinase 1 & $\mathbf{4 . 4 1 ^ { - }}$ & 3.46 \\
\cline { 2 - 4 } MAPK14 Mitogen activated protein kinase 14 & 0.92 & 1.32 \\
\cline { 2 - 4 } MÁPK3 & Mitogen activated protein kinase 3 & $\mathbf{5 . 7 8 ^ { - }}$ & $\mathbf{5 . 0 2}$ \\
\cline { 2 - 4 } MAPK8 Mitogen-activated protein kinase 8 & 2.99 & 2.28 \\
\hline
\end{tabular}

等

\begin{tabular}{|c|c|c|c|}
\hline \multicolumn{2}{|c|}{$\begin{array}{l}\text { T cells and co-stimulatory molecules } \\
\text { CDT4 }\end{array}$} & 3.32 & 7.75 \\
\hline $\begin{array}{l}\mathrm{CD} B \mathrm{~A} \\
\mathrm{FOXP3}\end{array}$ & $\begin{array}{l}\text { CD8a molecule } \\
\text { Forkheadbox P3 }\end{array}$ & $\begin{array}{l}\frac{5.96 " \dagger}{1.15} \\
15\end{array}$ & $\begin{array}{l}3.05 \\
0.29 \\
0.29\end{array}$ \\
\hline 040 & $\begin{array}{l}\text { Lutumolecule, IN F recepto } \\
\text { superfanily member } 5\end{array}$ & $11.64^{\circ}$ & \\
\hline & $\begin{array}{l}\text { CDL0 ligand } \\
\text { Cd80 molecule }\end{array}$ & $4.45^{*}$ & \\
\hline CD86 & CD86 molecule & \begin{tabular}{|l|l|l|l|l}
$20.48^{-1}$ \\
215
\end{tabular} & $\frac{21.85}{2.04}$ \\
\hline RAG & Recombination activating gene 1 & $\frac{2.13}{3.11}$ & \\
\hline
\end{tabular}

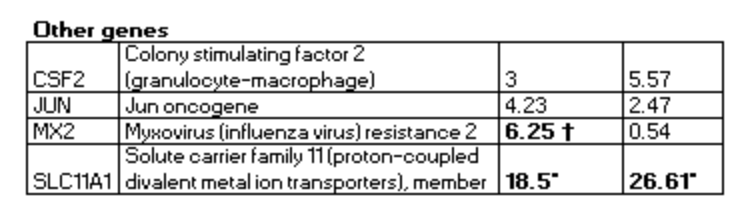

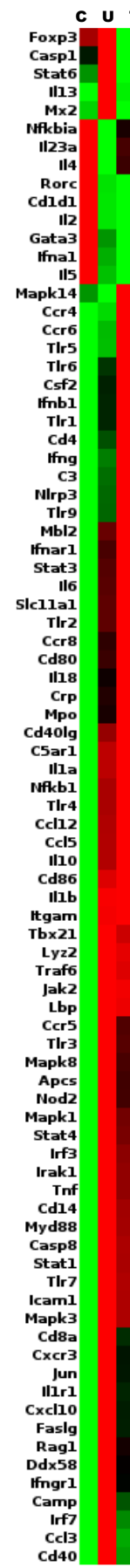

1

Downregulated genes

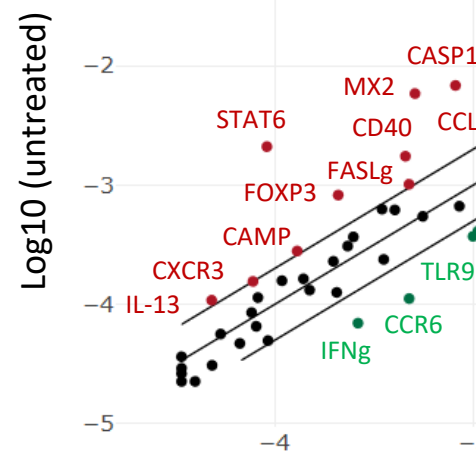

LIR1 $70 \%$

IRF7 CXCL10,0

TLRI C3

Upregulated genes

Log10 (sponge-treated)

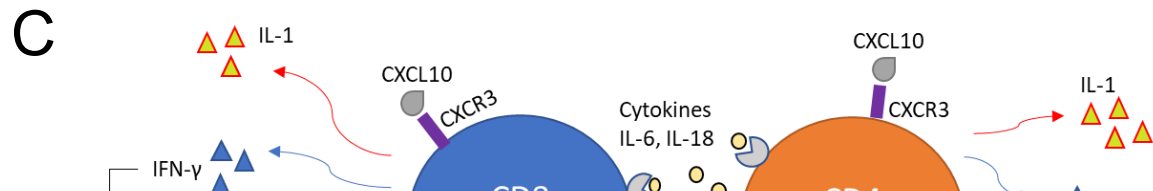

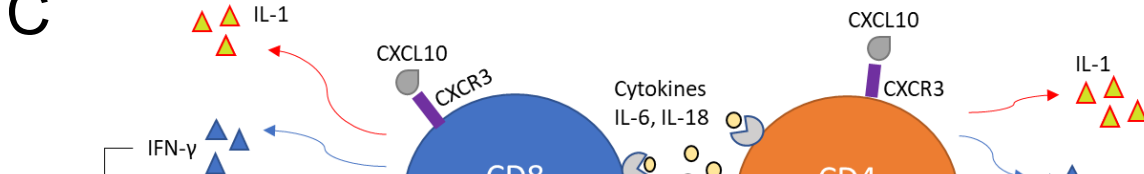

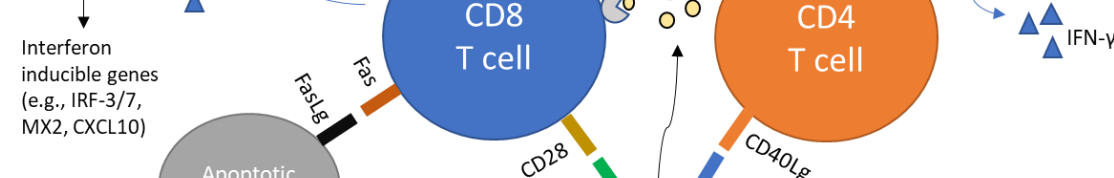

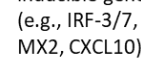
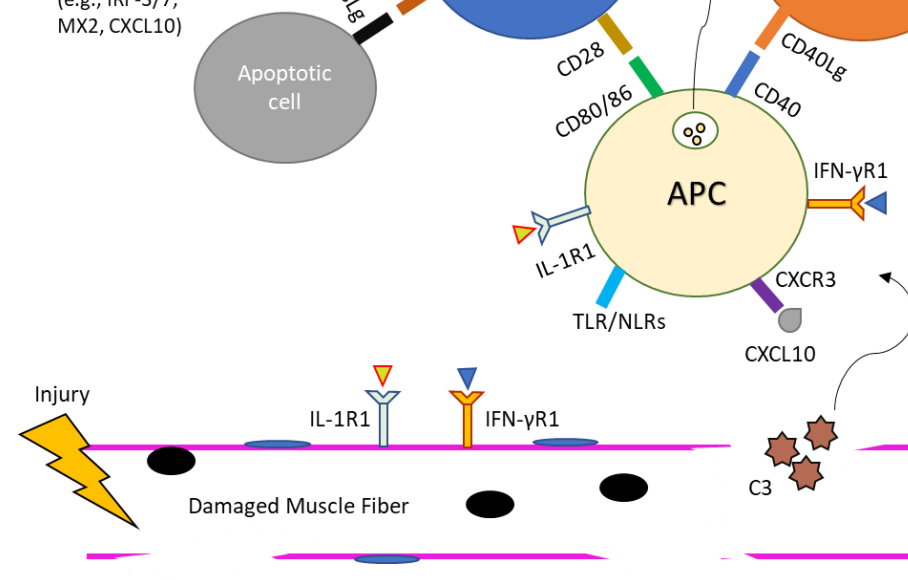

C3 है? 


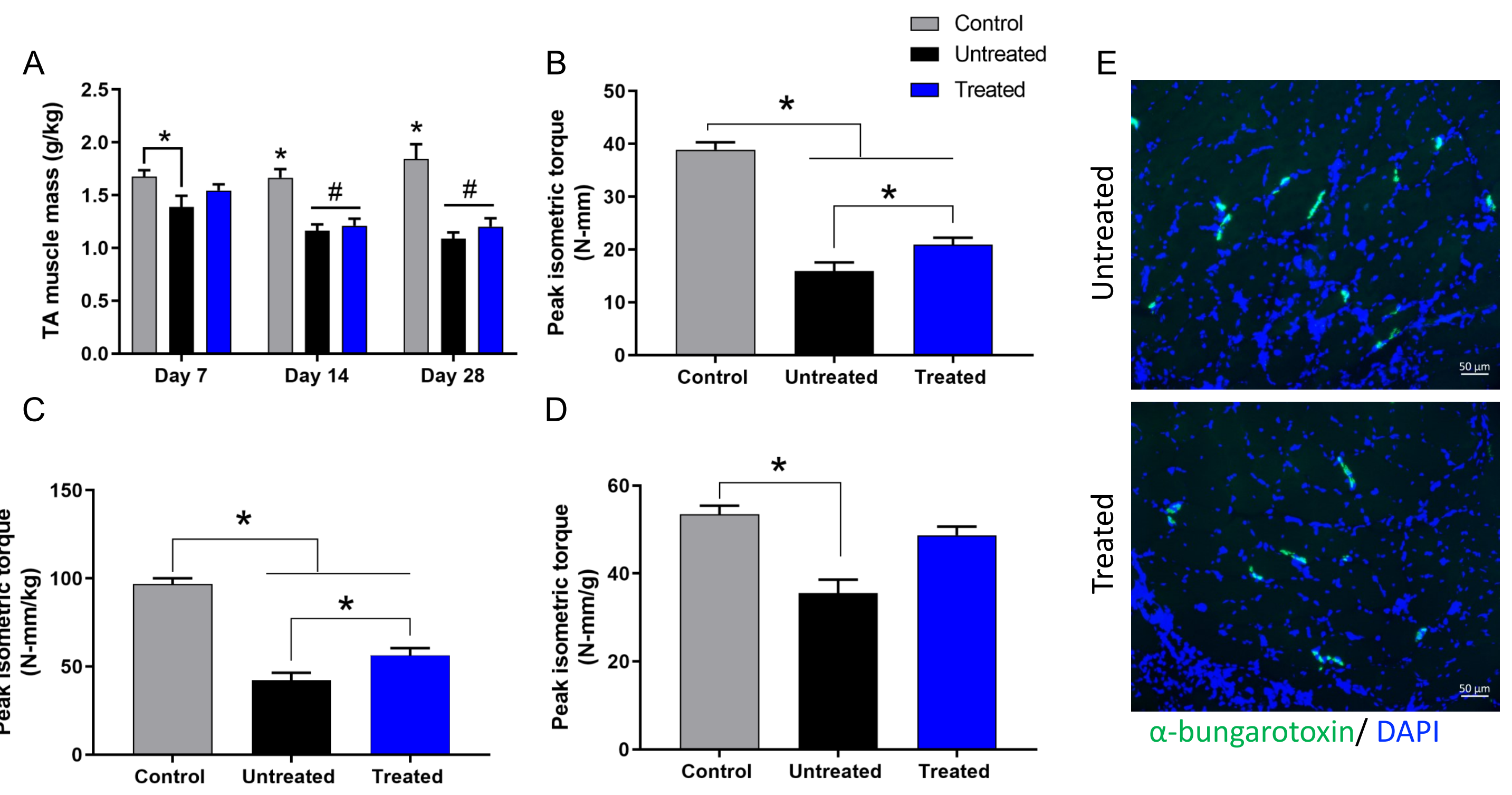

Fig. 6 


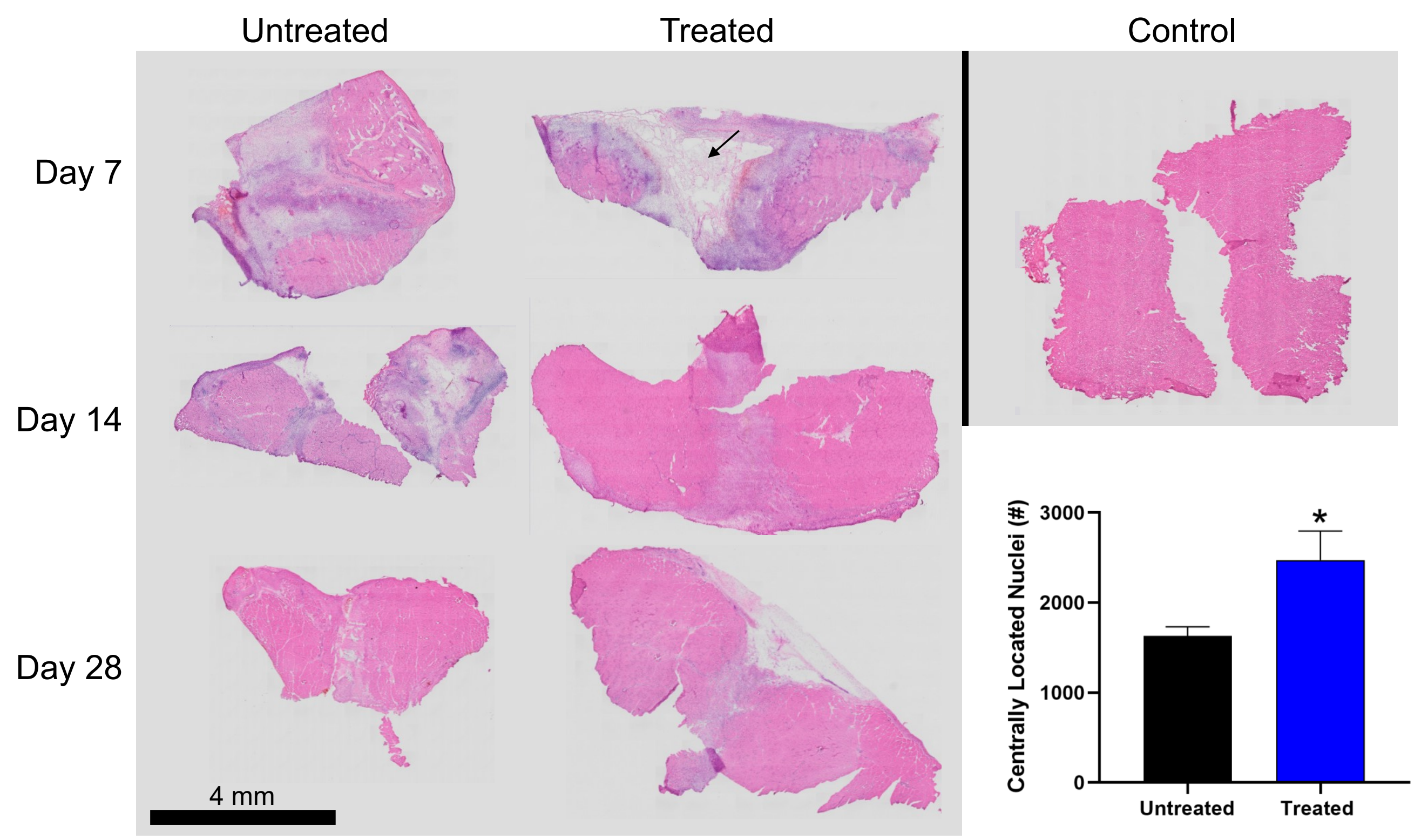

Fig. 7 


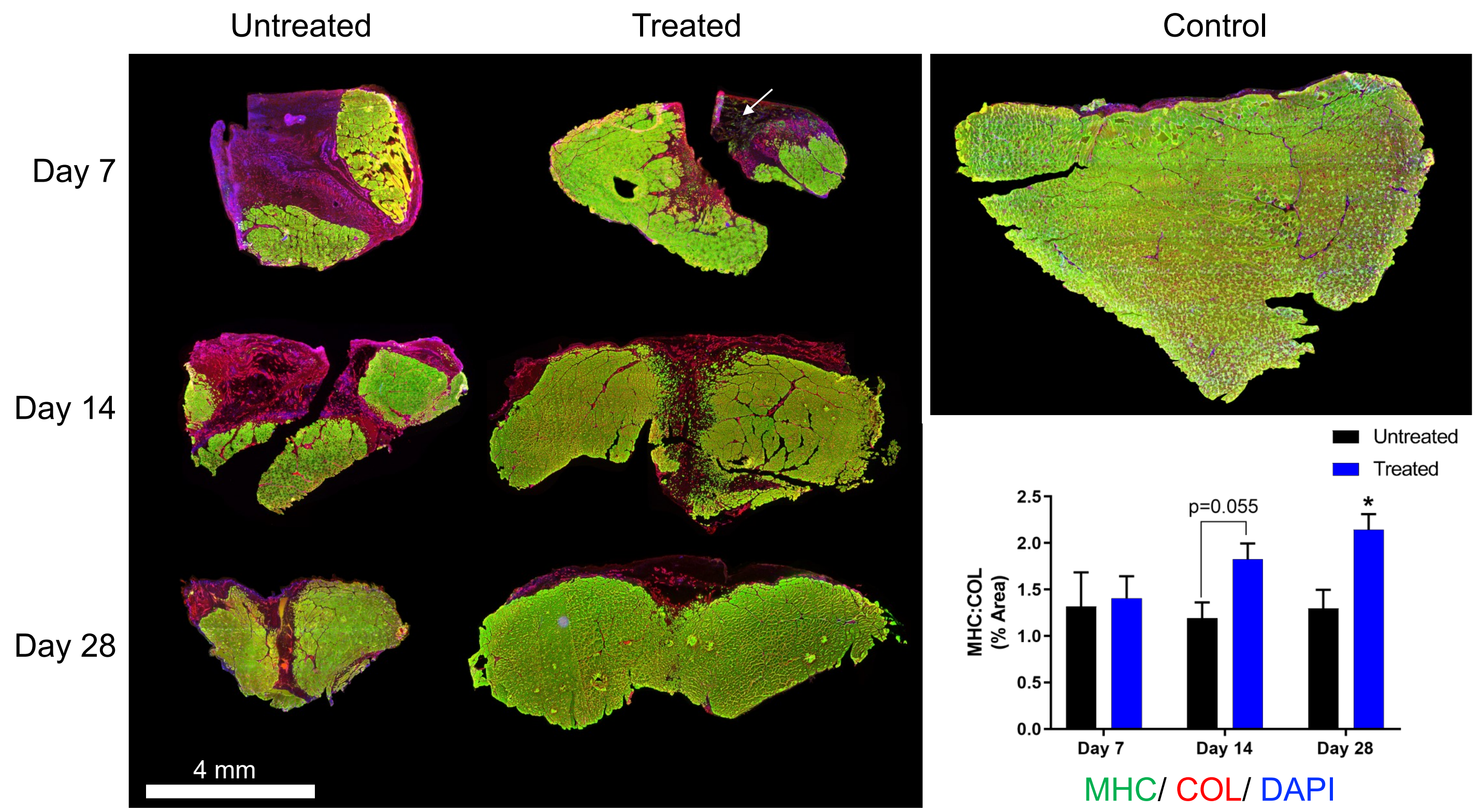

Fig. 8 


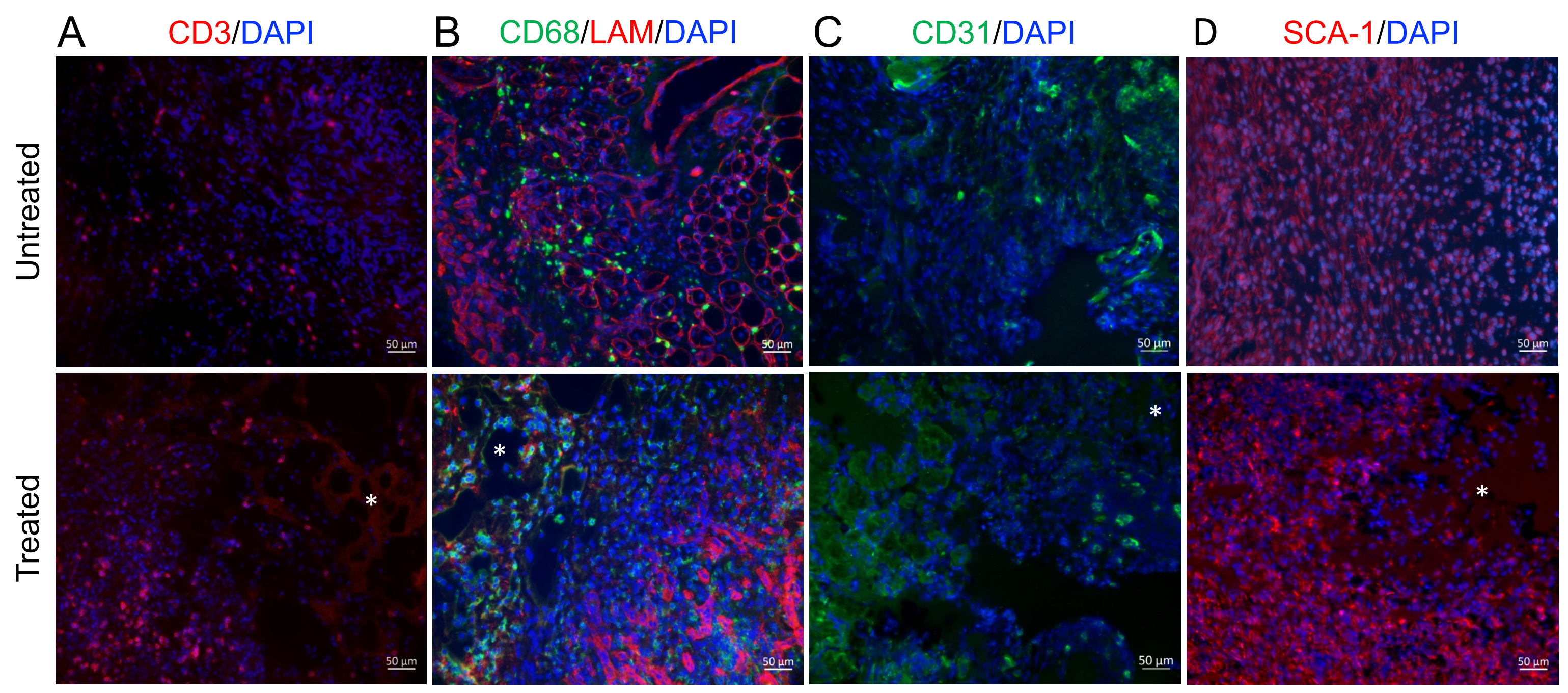

Fig. 9 

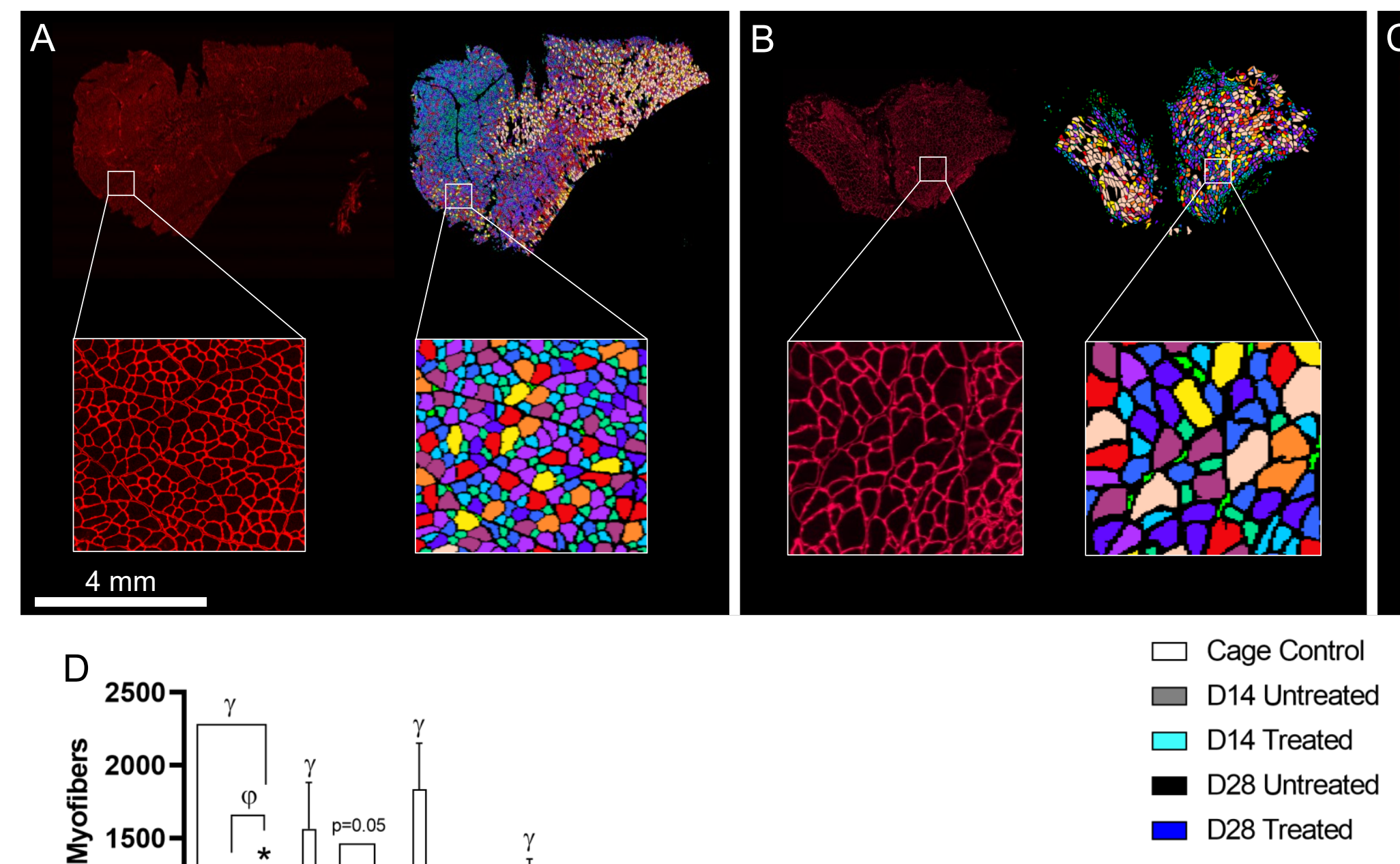

Fig. 10

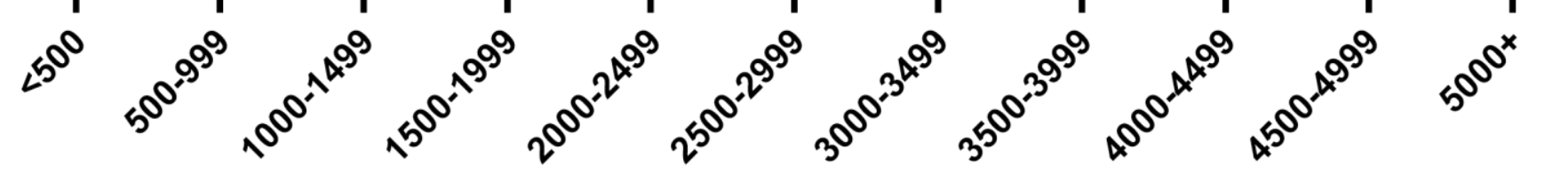

Cross-sectional area $\left(\mu \mathrm{m}^{2}\right)$
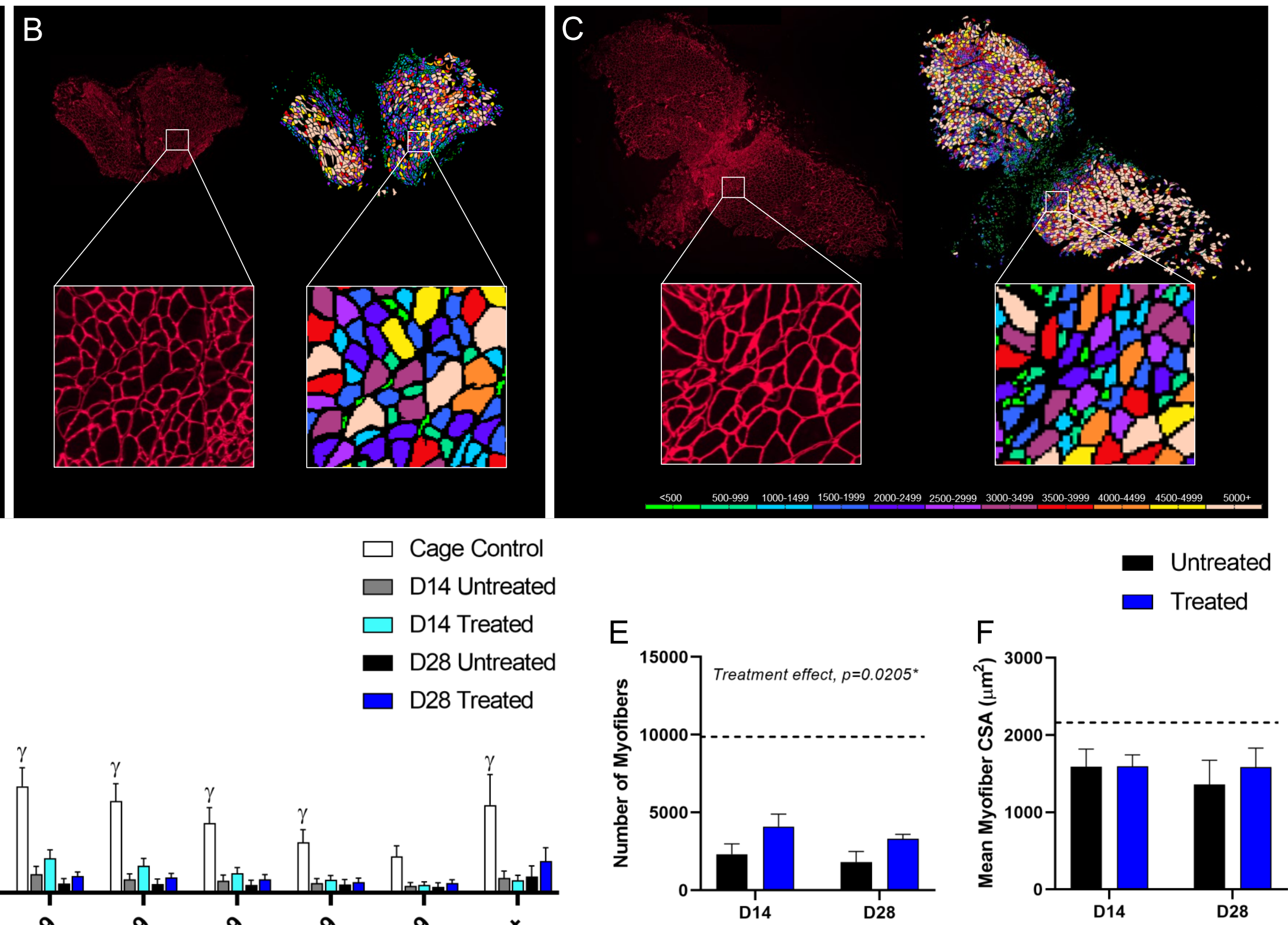

- Untreated 


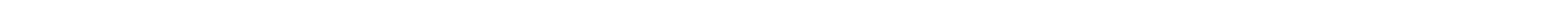




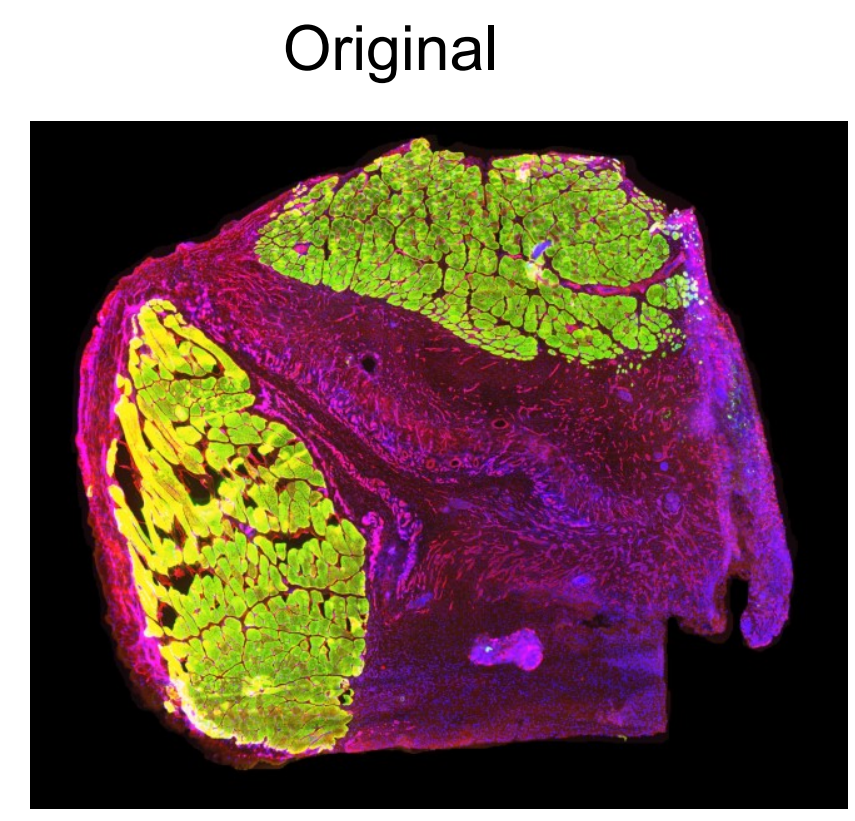

$\mathrm{MHC/} \mathrm{COL} / \mathrm{DAPI}$
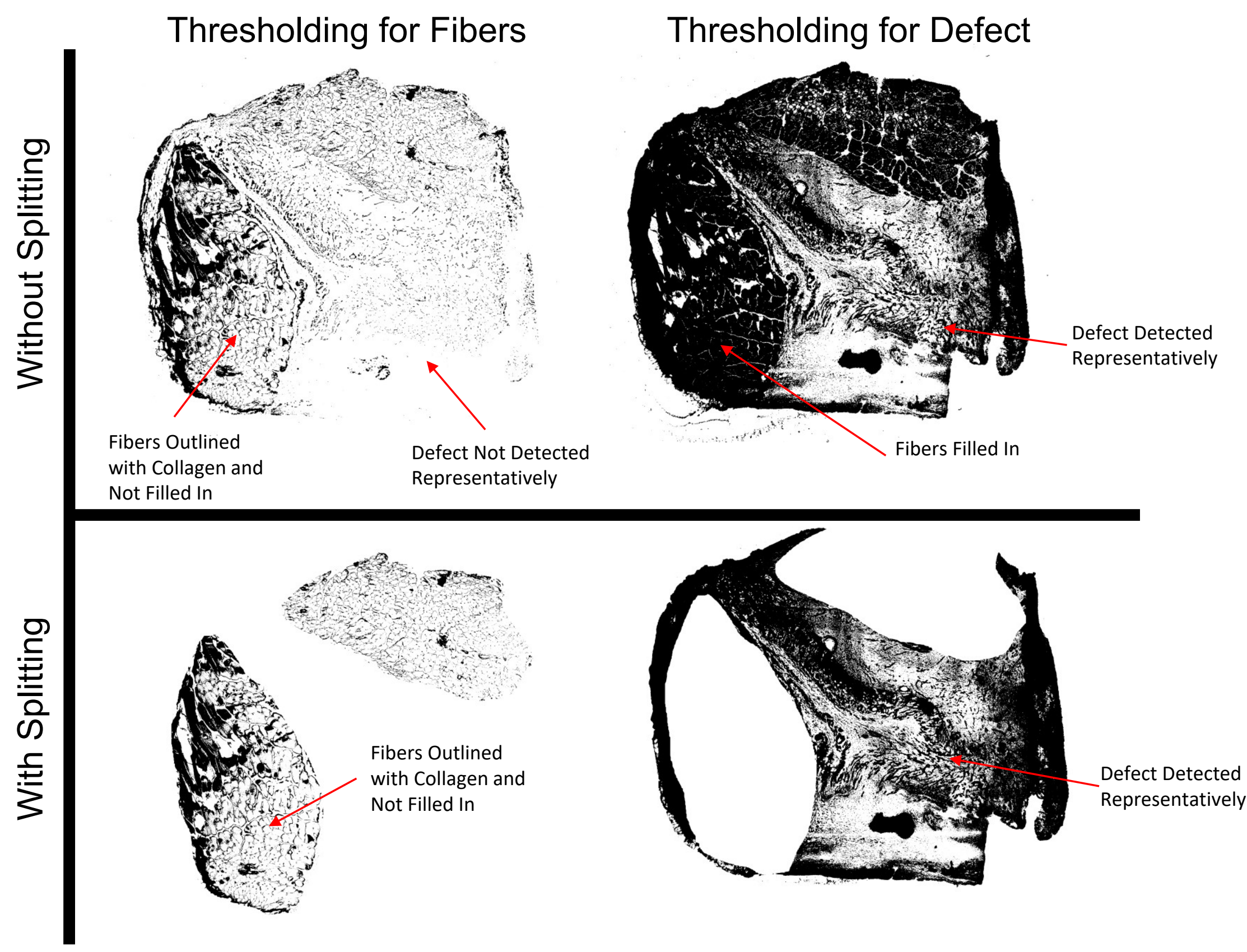


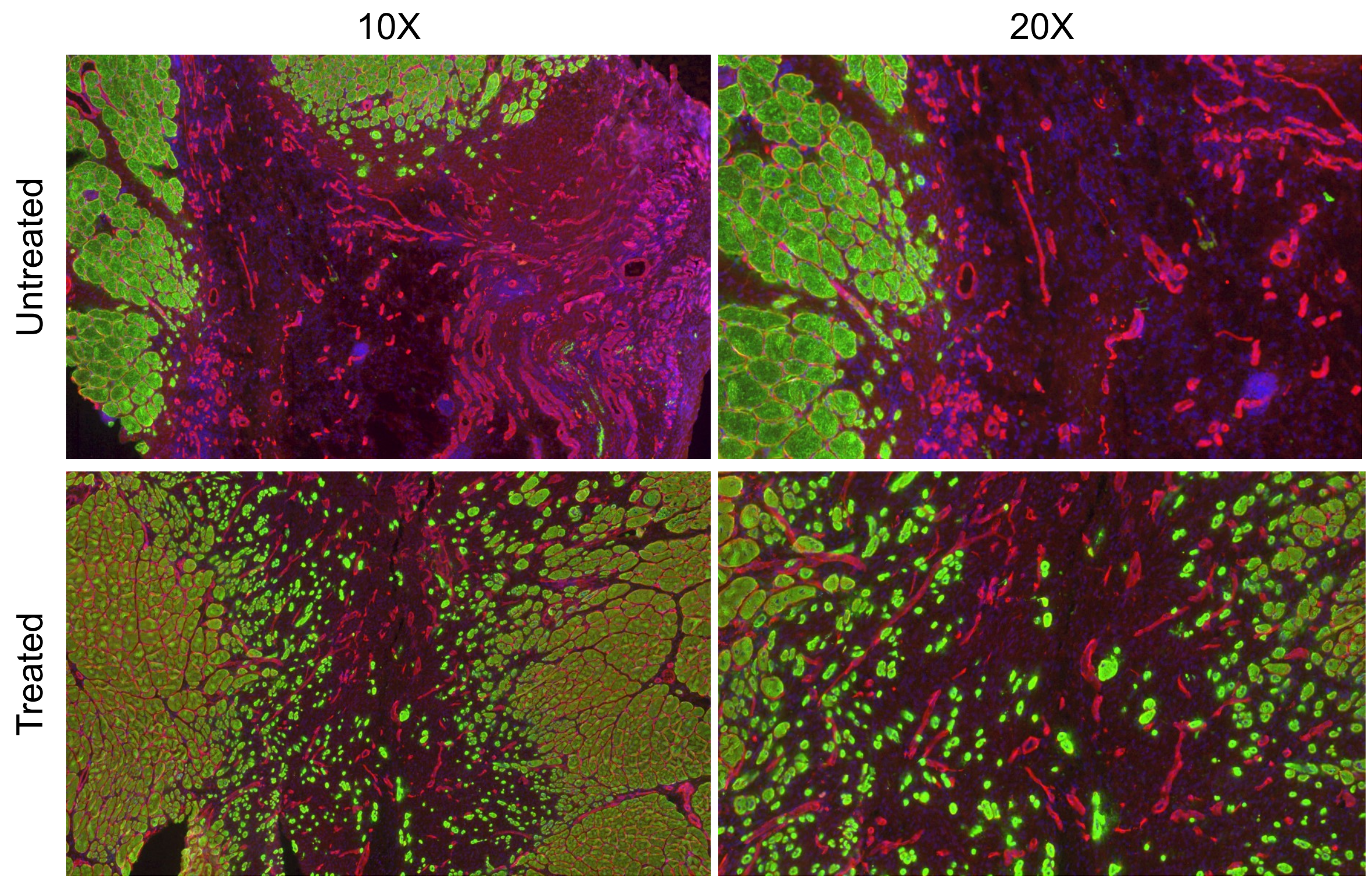

$\mathrm{MHC} / \mathrm{COL} / \mathrm{DAPI}$

Supplementary Fig. 2 
H\&E
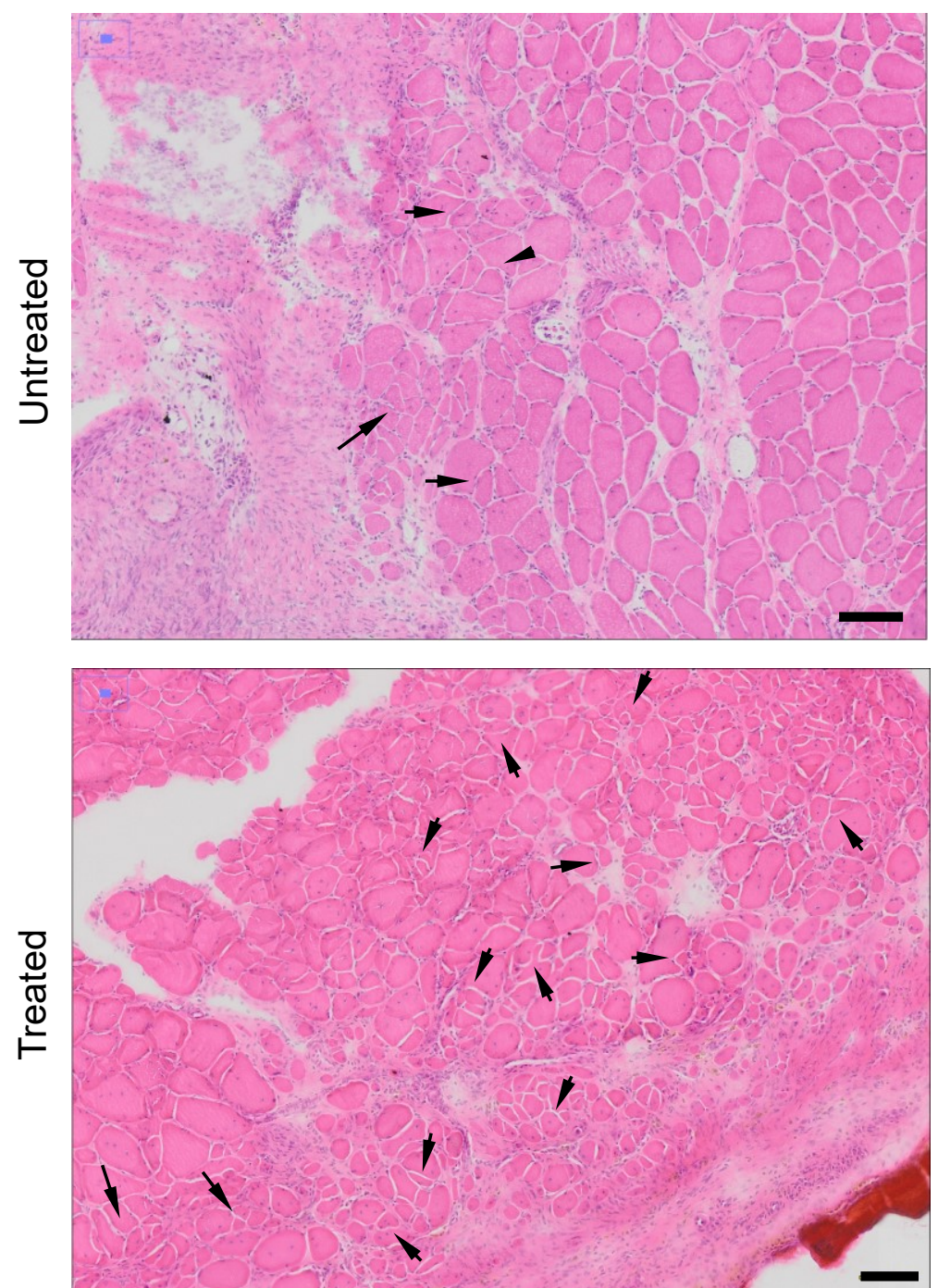

Type 1 / 2A / 2B
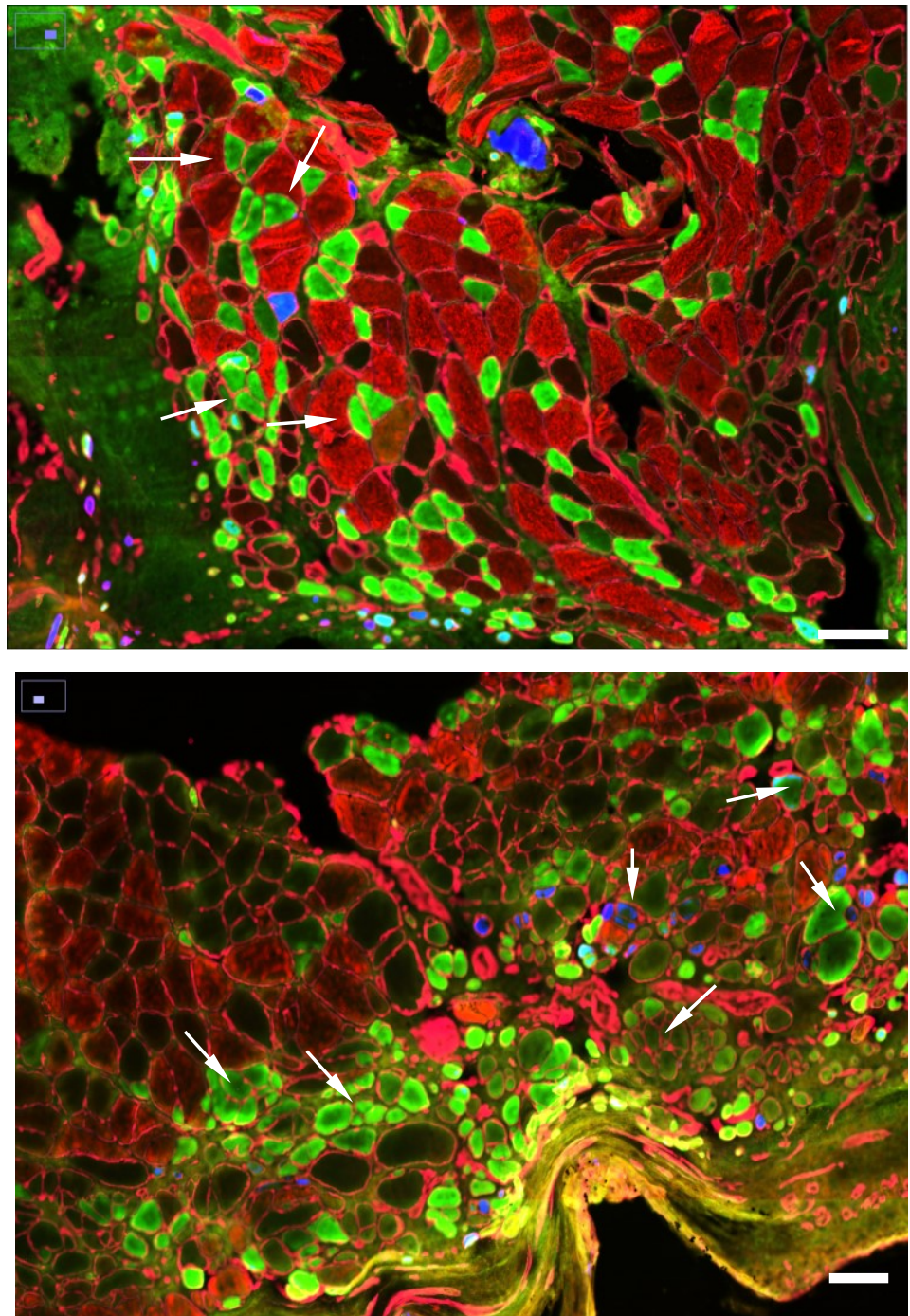

Scale bar $=100 \mu \mathrm{M}$

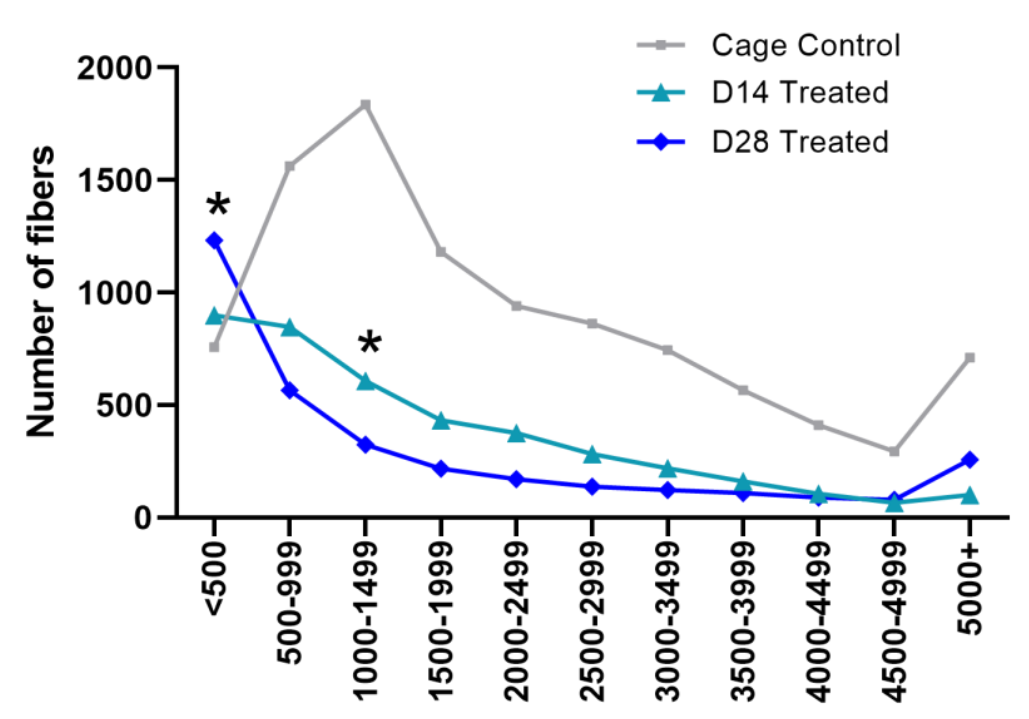

Supplementary Fig. 3 


\begin{tabular}{|c|c|c|c|}
\hline \multicolumn{4}{|c|}{ Table 1. Primers for qrtPCR } \\
\hline Gene & Forward Sequence & Reverse Sequence & Amplicon length, bp \\
\hline Pax7 & 5'-GCAGTCGGACCACATTCAC-3' & 5'-CGCACGACGGTTACTGAAC-3' & 155 \\
\hline MyoD & 5'-CGTGGCAGTGAGCACTACAG-3' & 5'-TGTAGTAGGCGGCGTCGTA-3' & 133 \\
\hline Myogenin & 5'-CTACAGGCCTTGCTCAGCTC-3' & 5'-GTTGGGACCAAACTCCAGTG-3' & 153 \\
\hline $\mathrm{eMHC}$ & 5'-TGGAGGACCAAATATGAGACG-3' & 5'-CACCATCAAGTCСТССАССТ-3' & 180 \\
\hline CTGF & 5'-CAAGCAGCTGGGAGAACTG-3' & 5'-ACAGGGTGCACCATCTTTG-3' & 127 \\
\hline COL1 & 5'-CTGGTGAACGTGGTGCAG-3' & 5'-GACCAATGGGACCAGTCAGA-3' & 123 \\
\hline TGFB1 & 5'-GTCAGACATTCGGGAAGCA-3' & 5'-CCAAGGTAACGCCAGGAAT-3' & 138 \\
\hline $18 S$ & 5'-GGCCCGAAGCGTTTACTT-3' & 5'-ACCTCTAGCGGCGCAATAC-3' & 173 \\
\hline
\end{tabular}

Table 1 


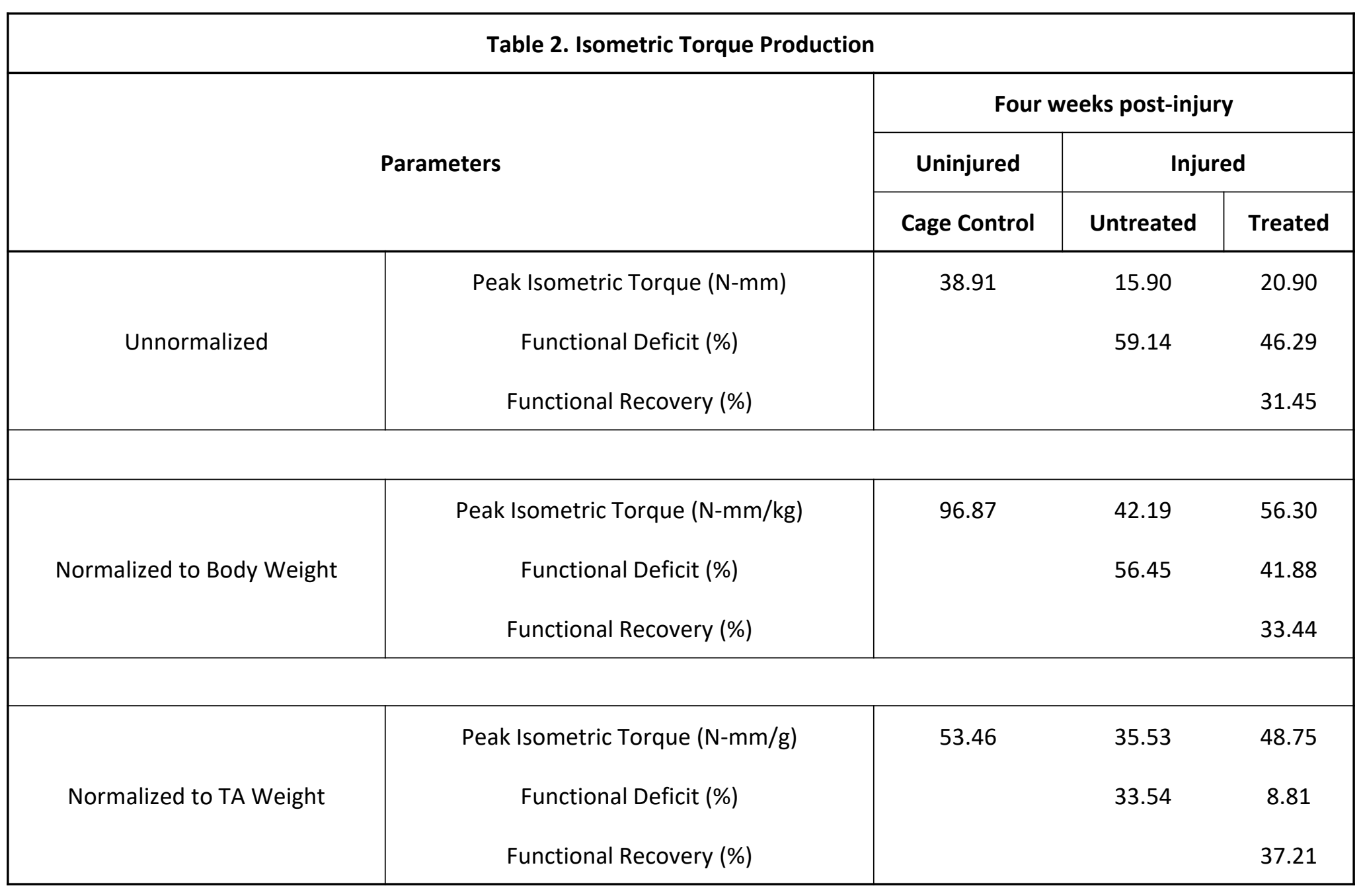

Table 2 